\title{
Biased Tracer Diffusion in Hard-Core Lattice Gases: Some Notes on the Validity of the Einstein Relation.
}

\author{
G.Oshanin ${ }^{1}$, O.Bénichou ${ }^{2}$, S.F.Burlatsky ${ }^{3}$ and M.Moreau ${ }^{1}$ \\ ${ }^{1}$ Laboratoire de Physique Théorique des Liquides, \\ Université Paris 6, 4 Place Jussieu, 75252 Paris, France \\ ${ }^{2}$ Laboratoire de Physique de la Matière Condensée, \\ Collège de France, 11 Place M.Berthelot, 75252 Paris Cedex 05, France \\ ${ }^{3}$ United Technologies Research Center, \\ United Technologies Corporation, \\ 411 Silver Lane, 129-21 East Hartford, CT 06108, USA
}

\begin{abstract}
In this presentation we overview some recent results on biased tracer diffusion in lattice gases. We consider both models in which the density of lattice gas particles is explicitly conserved and situations in which the lattice gas particles undergo continuous exchanges with a reservoir, which case is appropriate, e.g. to adsorbed monolayers in contact with the vapor phase. For all these models we determine, in some cases exactly and in other ones - using a certain decoupling approximation, the mean displacement of a tracer particle (TP) driven by a constant external force in a dynamical background formed by the lattice gas particles whose transition rates are symmetric. Evaluating the TP mean displacement explicitly we are able to define the TP mobility, which allows us to demonstrate that the Einstein relation between the TP mobility and the diffusivity generally holds, despite the fact that in some cases diffusion is anomalous. For models treated within the framework of the decoupling approximation, our analytical results are confirmed by Monte Carlo simulations. Perturbance of the lattice gas particles distribution due to the presence of a biased TP and the form of the particle density profiles are also discussed.
\end{abstract}




\section{Introduction.}

Transport in media with quenched or time-dependent disorder has received much interest due to the considerable fundamental and technological importance of the problem [1-8]. Transport processes underlay a variety of chromatographic separation techniques [4-6], extraction of oil from porous rocks [4-6], excitations quenching in amorphous solids and doped crystals $[6,7,9-12]$, conductivity of complex media and permeability of disordered membranes $[8,13]$. Molecular diffusion measurements in disordered media often serve as a tool in characterizing the internal geometry over a range of molecular and macroscopic length scales [4-7]. In addition, transport processes control the kinetics of chemical reactions in disordered media [9-12].

Following motion of individual particles in disordered media one most often encounters behavior characterized by a mean-square displacement $<\mathbf{R}^{2}(t)>$ which grows linearly in time,

$$
<\mathbf{R}^{2}(t)>\sim D t
$$

$D$ being the proportionality factor - the "diffusion" coefficient, which depends in a com-

plicated fashion on the peculiar features of the properties of the disordered medium. Such type of dynamics is ubiquitous due to its inherent relationship to the central-limit theorem, which makes it a paradigm of stochastic processes.

One the other hand, there has been growing evidence for other laws of random transport, both slower and enhanced relative to the time dependence in Eq.(1). Generally, one finds

$$
<\mathbf{R}^{2}(t)>\sim t^{\alpha}, \text { with } \alpha \neq 1
$$

Case with $\alpha<1$, known as the dispersive transport or subdiffusive regime, has been in the focus of many theoretical and experimental studies (see, e.g., Refs.[1-4,12]) and has been attributed, for instance, to random walks on fractal structures $[1-4,12]$ or to diffusion in presence of temporal traps $[1,12,14]$. A few stray examples of such a transport are time of flight measurements in amorphous semiconductors [14,15], defect diffusion in relaxation studies [14], excitation migration on the disordered array of donor centers 
(see, e.g. Ref.[16]), tracer diffusion in a linear array of convective rolls [17] or in lowdimensional lattice gases [18-21], or the so-called Sinai diffusion with extremely confined particle trajectories such that $<\mathbf{R}^{2}(t)>\sim \ln ^{4}(t)$ [22-24]. Enhanced diffusion regime, $\alpha>1$, is observed in turbulent diffusion $(\alpha=3)$, as well as in some other instances (see, e.g. Ref.[25]) for some review).

Suppose now that in the disordered medium in question, in which, in absence of any fields acting on the species whose motion we follow (say, tracer particles), we find that their mean displacement is zero, while the mean-square displacement $<\mathbf{R}^{2}(t)>$ obeys Eq.(1) with $D$ being some function of disorder or the anomalous diffusion law in Eq.(2). Suppose next that we manage to bias somehow the particle motion, say, by charging the tracer particles only and putting the whole system in constant electric field $E$. This situation is, of course, quite interesting in its own right and is appropriate to charge transfer or dynamic directed percolation in time-dependent inhomogeneous medium [26-29], such as, e.g. solid protonic conductors [31], oil-continuous microemulsions [32-35] or polymer electrolytes [36-38]. In such a situation, the tracer particles will move preferentially in the direction of the field and will attain a non-zero mean displacement and a non-zero mean velocity $V(t)$. One may then define the time-dependent tracer particle mobility as

$$
\mu(t)=\lim _{E \rightarrow 0} \frac{V(t)}{E}
$$

while the "diffusivity" $D(t)$ can be extracted from the behavior in absence of the field (Eqs.(1) or (2)) via:

$$
D(t)=\frac{<\mathbf{R}^{2}(t)>}{2 d t}
$$

where $d$ is the spatial dimension of the system.

Now, the question is whether the mobility $\mu(t)$, calculated from the tracer particle mean displacement in the presence of an external electric field, and the diffusivity $D(t)$, Eq.(4), deduced from the tracer particle mean-square displacement in the absence of the field, obey the generalized Einstein relation of the form

$$
\mu(t)=\beta D(t)
$$


where $\beta$ denotes the reciprocal temperature?

The answer is trivially positive, of course, for isolated non-interacting particles executing conventional Brownian motion. For particle diffusion in disordered medium the validity of the relation in Eq.(5) is much less evident. On the one hand, it has been found that Eq.(5) holds for the tracer particle diffusion in a 1D hard-core gas when the onedimensional lattice is a ring of a finite length [39] but also for infinite 1D lattices with non-conserved [40] and conserved particles number [41-43]. Remarkably, in the latter case Eq.(5) holds for $t$ sufficiently large despite the fact that both the tracer particle mobility and the diffusivity are not constant as $t \rightarrow \infty$ but all vanish [41-43]. As well, the validity of the Einstein relation has been corroborated for the charge carriers in semiconductors [44] and for polymeric systems in the sub-diffusive regime [45, 46]. On the other hand, it is well known that the Einstein relation is violated in some physical situations; for instance, it is not fulfilled for Sinai diffusion [22-24] or diffusion on percolation clusters, due to effects of strong temporal trapping in the dangling ends, as well as for the Scher-Lax-Montroll model of anomalous random walk [47] (see also Refs.[48] and [49] for some other examples). Hence, in principle, it is not a priori clear whether Eq.(5) should be valid in any case.

In this presentation we overview some recent results on the validity of the generalized Einstein relation in Eq.(5) for tracer particle (TP) diffusion in dynamical disordered environments, as exemplified here by non-interacting lattice gases of hard-core particles. The paper is structured as follows: In Section 2 we introduce the model, basic notations, write down dynamic equations describing the time evolution of the system and discuss the way of their solution. In Section 3 we first recall the properties of an unbiased TP diffusion in a one-dimensional hard-core lattice gas. Next, we turn to the case of a biased TP in an inert lattice gas and present explicit results describing the TP mean displacement and the mobility. We demonstrate then that the Einstein relation in Eq.(5) holds exactly despite the fact that in this case diffusion is anomalous. Further on, in Section 4 we consider the case when the initial particle distribution on a one-dimensional lattice is inhomogeneous and characterized by an $S$-shape, "shock"-like density profile. We find that when the TP is attracted towards the particle phase with higher density, at a certain critical value of 
the constant attraction force the TP will not move, on average. Assuming the validity of the Einstein relation, we find the second moment of the TP displacement. This heuristic analytical result is confirmed numerically which signifies that the Einstein relation holds apparently for such an inhomogeneous situation. Next, in Sections 5 and 6 we analyse the forms of the biased TP terminal velocity in one-, two- and three-dimensional hard-core lattice gases undergoing continuous particles exchanges with a reservoir. We determine the TP mobility and assuming the validity of the Einstein relation, obtain the TP diffusion coefficient $D$ in the unbiased case. Results for one-dimensional systems are confirmed by numerical Monte Carlo simulations. We also show that in two- and three-dimensional cases in the conserved particle density limit (when particle exchanges with the reservoir are forbidden) our results for $D$ coincide with classical predictions of Nakazato and Kitahara [52], which are known to be in a very good agreement with the numerical data [51]. In Section 7 we present an exact solution for the biased TP mean displacement in a twodimensional lattice gas containing a single unoccupied site. On comparing our results with earlier results by Brummelhuis and Hilhorst [55] obtained for the unbiased TP dynamics, we infer that also in this case, in which the TP diffusion is anomalously, logarithmically confined, the Einstein relation does hold exactly. Finally, we conclude in Section 8 with a brief summary of results and discussion.

\section{The model and basic equations.}

Consider a $d$-dimensional regular hypercubic lattice of spacing $\sigma$ each site of which is brought in contact with a reservoir containing identic, electrically neutral particles - a vapor phase (Fig.1), maintained at a constant pressure. We suppose next that the reservoir particles may be created (adsorbed) on any vacant site at a fixed rate $f / \tau^{*}$, which rate depends on the reservoir pressure and the energy gain due to the "creation" event. Further on, the particles may move randomly along the lattice by hopping at a rate $1 / 2 d \tau^{*}$ to any of $2 d$ neighboring lattice sites, which process is constrained by hard-core exclusion preventing multiple occupancy of any of the sites. Lastly, the particles may spontaneously 
disappear (desorb) from the lattice at rate $g / \tau^{*}$, which is dependent on the barrier against desorption. Both $f$ and $g$ are site and environment independent.

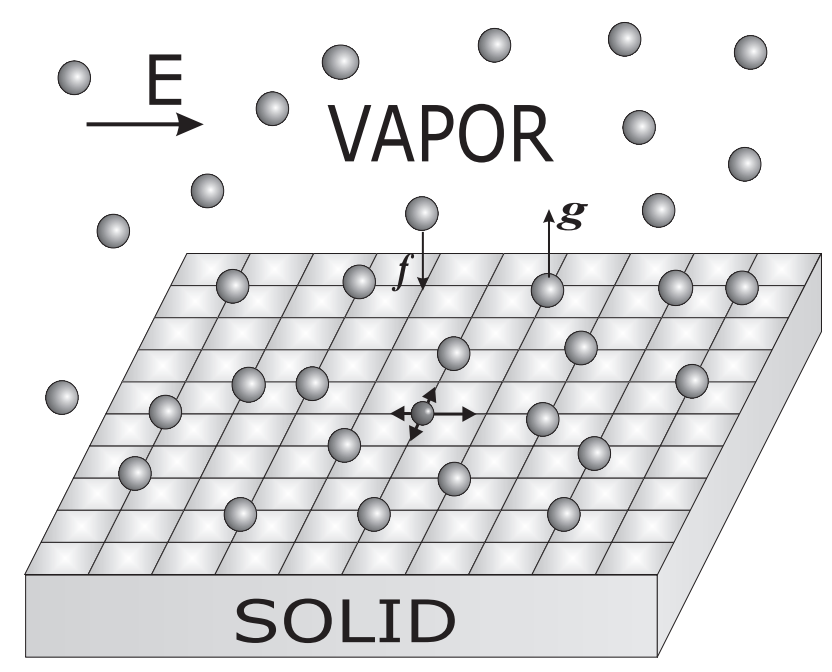

Figure 1: Two-dimensional realization of the model: Adsorbed monolayer in contact with a vapor phase. Grey spheres denote the monolayer (vapor) particles; the smaller black sphere stands for the driven tracer particle.

To describe the occupancy of lattice sites, we introduce a time-dependent variable $\eta(\mathbf{R})$, which may assume two values:

$$
\eta(\mathbf{R})=\left\{\begin{array}{l}
1, \text { if the site } \mathbf{R} \text { is occupied } \\
0, \text { if the site } \mathbf{R} \text { is empty. }
\end{array}\right.
$$

Note that the local variable $\eta(\mathbf{R})$ can change its value due to creation of particles (adsorbtion), desorption and random hopping events. Note also that random hopping events do conserve the total number of adsorbed particles and hence, the average particle density $\rho_{s}(t)$. On the other hands, creation/desorption processes do change $\eta(\mathbf{R})$ locally such that the total number of particles on the lattice is not explicitly conserved when such two processes are allowed. However, the mean particle density, $\rho_{s}(t)=\langle\eta(\mathbf{R})\rangle$, approaches as $t \rightarrow \infty$ a constant value

$$
\rho_{s}=\frac{f}{f+g}
$$


This relation is well known and represents the customary Langmuir adsorption isotherm [50]. We finally remark that in the analysis of the stationary-state behavior, we can always turn to the conserved particles number limit by setting $f$ and $g$ equal to zero and keeping their ratio fixed, i.e. supposing that $f / g=\rho_{s} /\left(1-\rho_{s}\right)$. This limit will correspond to the model of biased tracer diffusion in a hard-core lattice gas with fixed particles density $\rho_{s}$, and will allow us to check our analytical predictions against some already known results $[51-55]$.

Further on, at $t=0$ we introduce at the lattice origin an extra hard-core particle, whose motion we would like to follow and which will be called in what follows as the tracer particle - the TP; position of this particle at time $t$ is denoted as $\mathbf{R}_{t r}$. We stipulate that the TP is different from other particles in two aspects: first, it can not disappear from the lattice and second, it is subject to some external driving force, which favors its jumps into a preferential direction. Physically, such a situation may be realized, for instance, if this only particle is charged and the system is subject to a uniform electric field $\mathbf{E}$. We suppose here, for simplicity of exposition, that the external force $\mathbf{E}$ is oriented according to the unit vector $\mathbf{e}_{\mathbf{1}}$.

The dynamics of the biased TP is defined in the usual fashion [18-21]: We suppose that the TP, which occupies the site $\mathbf{R}_{t r}$ at time $t$, waits an exponentially distributed time with mean $\tau$, and then attempts to hop onto one of $2 d$ neighboring sites, $\mathbf{R}_{t r}+\mathbf{e}_{\boldsymbol{\nu}}$, where $\mathbf{e}_{\boldsymbol{\nu}}$ are $2 d$ unit vectors of the hypercubic lattice. In what follows we adopt the notation $\nu=\{ \pm 1, \pm 2 \ldots, \pm d\}$, where $\mathbf{e}_{\mathbf{1}}$ will denote the direction of the external force $\mathbf{E}$. Next, the jump direction is chosen according to the probablity $p_{\nu}$, which obeys:

$$
p_{\nu}=\frac{\exp \left[\frac{\beta}{2}\left(\mathbf{E} \cdot \mathbf{e}_{\boldsymbol{\nu}}\right)\right]}{\sum_{\mu} \exp \left[\frac{\beta}{2}\left(\mathbf{E} \cdot \mathbf{e}_{\boldsymbol{\mu}}\right)\right]}
$$

where $\beta$ is the reciprocal temperature, $(\mathbf{E} \cdot \mathbf{e})$ stands for the scalar product, the charge of the TP is set equal to unity and the sum with the subscript $\mu$ denotes summation over all possible orientations of the vector $\boldsymbol{e}_{\mu}$; that is, $\mu=\{ \pm 1, \pm 2 \ldots, \pm d\}$.

After the jump direction is chosen, the TP attempts to hop onto the target site. The 
hop is instantaneously fulfilled if the target site is vacant at this moment of time; otherwise, i.e., if the target site is occupied by any adsorbed particle, the jump is rejected and the $\mathrm{TP}$ remains at its position.

To close this subsection, several comments on the relevance of the model to some physical situations are in order. As we have already remarked, in the conserved particles number limit the model under study reduces to the model of biased tracer diffusion in hard-core lattice gases, which is related to a wide variety of physical situations, including, for example, diffusion of interstitials in crystals. A detailed review can be found in Ref.[51]. In two-dimensions, in the non-conserved particles number case it describes tracer diffusion in adsorbed monolayers in contact with the vapor phase [56] and allows to determine, for instance, the analog of the Stokes formula for such monolayers and consequently, to elucidate their intrinsic frictional properties. On the other hand, the two-dimensional case can be thought of as some simplified picture of the stagnant layers emerging in liquids that are in contact with a solid body. It is well-known (see, e.g. Ref.[65]) that liquids in the close vicinity of a solid interface - at distances within a few molecular diameters do possess completely different physical properties compared to those of the bulk phase. In this stagnant region, in which an intrinsically disordered liquid phase is spanned by and interacts with the ordering potential of the solid, the liquid's viscosity is drastically enhanced and transport processes (related, say, to biased diffusion of charged carriers in solutions) are substantially hindered. Thus our model can be viewed as a two-level approximate model of this challenging physical system, in which the reservoir mimics the bulk fluid phase with very rapid transport, while the adsorbed monolayer (particles on a 2D lattice) represents the stagnant layer emerging on the solid-liquid interface. Lastly, the model under study represents a novel example [29] of the so-called "dynamic percolation" models (see, e.g. Refs.[26-28] and references therein), invoked to describe transport processes in many situations with dynamical disorder. In this context, the lattice gas particles can be thought of as representing some fluctuating environment, which hinders the motion of an impure molecule - the TP, which might be, for example, a charge carrier. A salient feature of this model, which makes it different from the previously proposed models of dynamic 
percolative environments, is that we include the hard-core interactions between the "environment" particles and the TP, such that the latter may itself influence the dynamics of the environment.

\subsection{Evolution equations.}

Now, we derive the evolution equations in a general, $d$-dimensional case. We begin by introducing some auxiliary definitions. Let $\eta \equiv\{\eta(\mathbf{R})\}$ denote the entire set of the occupation variables, which defines the instantaneous configuration of the lattice-gas particles at time moment $t$. Next, let $P\left(\mathbf{R}_{\mathrm{tr}}, \eta ; t\right)$ stand for the joint probability of finding at time $t$ the TP at the site $\mathbf{R}_{\operatorname{tr}}$ and all adsorbed particles in the configuration $\eta$. Then, denoting as $\eta^{\mathbf{r}, \nu}$ a configuration obtained from $\eta$ by the Kawasaki-type exchange of the occupation variables of two neighboring sites $\mathbf{r}$ and $\mathbf{r}+\mathbf{e}_{\boldsymbol{\nu}}$, and as $\hat{\eta}^{\mathbf{r}}$ - a configuration obtained from the original $\eta$ by the replacement $\eta(\mathbf{r}) \rightarrow 1-\eta(\mathbf{r})$, which corresponds to the Glauber-type flip of the occupation variable due to the creation/desorption events, we have that the time evolution of the configuration probability $P\left(\mathbf{R}_{\mathbf{t r}}, \eta ; t\right)$ obeys the following master equation:

$$
\begin{aligned}
& \partial_{t} P\left(\mathbf{R}_{\mathbf{t r}}, \eta ; t\right)=\frac{1}{2 d \tau^{*}} \sum_{\mu=1}^{d} \sum_{\mathbf{r} \neq \mathbf{R}_{\mathbf{t r}}-\mathbf{e}_{\mu}, \mathbf{R}_{\mathbf{t r}}}\left\{P\left(\mathbf{R}_{\mathbf{t r}}, \eta^{\mathbf{r}, \mu} ; t\right)-P\left(\mathbf{R}_{\mathbf{t r}}, \eta ; t\right)\right\} \\
+ & \frac{1}{\tau} \sum_{\mu} p_{\mu}\left\{\left(1-\eta\left(\mathbf{R}_{\mathrm{tr}}\right)\right) P\left(\mathbf{R}_{\mathrm{tr}}-\mathbf{e}_{\boldsymbol{\mu}}, \eta ; t\right)-\left(1-\eta\left(\mathbf{R}_{\mathrm{tr}}+\mathbf{e}_{\boldsymbol{\mu}}\right)\right) P\left(\mathbf{R}_{\mathrm{tr}}, \eta ; t\right)\right\} \\
+ & \frac{g}{\tau^{*}} \sum_{\mathbf{r} \neq \mathbf{R}_{\mathrm{tr}}}\left\{(1-\eta(\mathbf{r})) P\left(\mathbf{R}_{\mathbf{t r}}, \hat{\eta}^{\mathbf{r}} ; t\right)-\eta(\mathbf{r}) P\left(\mathbf{R}_{\mathbf{t r}}, \eta ; t\right)\right\} \\
+ & \frac{f}{\tau^{*}} \sum_{\mathbf{r} \neq \mathbf{R}_{\mathrm{tr}}}\left\{\eta(\mathbf{r}) P\left(\mathbf{R}_{\mathrm{tr}}, \hat{\eta}^{\mathbf{r}} ; t\right)-(1-\eta(\mathbf{r})) P\left(\mathbf{R}_{\mathbf{t r}}, \eta ; t\right)\right\} .
\end{aligned}
$$

The mean velocity $V(t)$ of the TP can be obtained by multiplying both sides of Eq.(8) by $\left(\mathbf{R}_{\mathrm{tr}} \cdot \mathbf{e}_{\mathbf{1}}\right)$ and summing over all possible configurations $\left(\mathbf{R}_{\mathbf{t r}}, \eta\right)$. This results in the following exact equation determining the TP velocity:

$$
V(t) \equiv \frac{d}{d t} \sum_{\mathbf{R}_{\mathrm{tr}}, \eta}\left(\mathbf{R}_{\mathrm{tr}} \cdot \mathbf{e}_{\mathbf{1}}\right) P\left(\mathbf{R}_{\mathrm{tr}}, \eta ; t\right)=\frac{\sigma}{\tau}\left\{p_{1}\left(1-k\left(\mathbf{e}_{\mathbf{1}} ; t\right)\right)-p_{-1}\left(1-k\left(\mathbf{e}_{-\mathbf{1}} ; t\right)\right)\right\},
$$


where

$$
k(\boldsymbol{\lambda} ; t) \equiv \sum_{\mathbf{R}_{\mathrm{tr}}, \eta} \eta\left(\mathbf{R}_{\mathrm{tr}}+\boldsymbol{\lambda}\right) P\left(\mathbf{R}_{\mathrm{tr}}, \eta ; t\right)
$$

is the probability of having at time $\mathrm{t}$ a lattice gas particle at position $\boldsymbol{\lambda}$, defined in the frame of reference moving with the TP. In other words, $k(\boldsymbol{\lambda} ; t)$ can be thought of as being the particle density profile as seen from the moving TP.

Equation (9) signifies that the velocity of the TP is dependent on the lattice gas particles density in the immediate vicinity of the tracer. If the lattice gas is perfectly stirred, or, in other words, if $k(\boldsymbol{\lambda} ; t)=\rho_{s}$ everywhere, (which implies immediate decoupling of $\mathbf{R}_{\mathrm{tr}}$ and $\eta$ ), one would obtain from Eq.(9) a trivial mean-field result

$$
V^{(0)}=\left(p_{1}-p_{-1}\right)\left(1-\rho_{s}\right) \frac{\sigma}{\tau}
$$

which states that the only effect of the medium on the TP dynamics is that its jump time $\tau$ is merely renormalized by a factor $\left(1-\rho_{s}\right)^{-1}$, which represents the inverse concentration of voids; note that then $\left(1-\rho_{s}\right) / \tau$ defines simply the mean frequency of successful jump events.

However, the situation appears to be more complicated and, as we proceed to show, $k(\boldsymbol{\lambda} ; t)$ is different from the equilibrium value $\rho_{s}$ everywhere, except for $|\boldsymbol{\lambda}| \rightarrow \infty$. This means that the TP strongly perturbs the particles distribution on the lattice - it is no longer uniform and some non-trivial density profiles emerge.

Now, in order to calculate the instantaneous mean velocity of the TP we have to determine the mean particles density at the neighboring to the TP sites $\mathbf{R}_{\mathbf{t r}}+\mathbf{e}_{ \pm \mathbf{1}}$, which requires, in turn, computation of the density profile $k(\boldsymbol{\lambda} ; t)$ for arbitrary $\boldsymbol{\lambda}$. The latter can be found from the master equation (8) by multiplying both sides by $\eta\left(\mathbf{R}_{\mathbf{t r}}\right)$ and performing the summation over all configurations $\left(\mathbf{R}_{\mathbf{t r}}, \eta\right)$. In doing so, we find that these equations are not closed with respect to $k(\boldsymbol{\lambda} ; t)$, but are coupled to the third-order correlations,

$$
T\left(\boldsymbol{\lambda}, \mathbf{e}_{\boldsymbol{\nu}} ; t\right)=\sum_{\mathbf{R}_{\mathbf{t r},}, \eta} \eta\left(\mathbf{R}_{\mathbf{t r}}+\boldsymbol{\lambda}\right) \eta\left(\mathbf{R}_{\mathbf{t r}}+\mathbf{e}_{\boldsymbol{\mu}}\right) P\left(\mathbf{R}_{\mathbf{t r}}, \eta ; t\right)
$$

In turn, if we proceed further to the third-order correlations, we find that these are coupled respectively to the fourth-order correlations. Consequently, in order to compute $V(t)$, one 
faces the problem of solving an infinite hierarchy of coupled equations for the correlation functions. Here we resort to the simplest non-trivial closure of the hierarchy in terms of $k(\boldsymbol{\lambda} ; t)$, which has been first proposed in Ref.[41], and represent $T\left(\boldsymbol{\lambda}, \mathbf{e}_{\boldsymbol{\nu}} ; t\right)$ as

$$
\begin{aligned}
& \sum_{\mathbf{R}_{\mathbf{t r}}, \eta} \eta\left(\mathbf{R}_{\mathrm{tr}}+\boldsymbol{\lambda}\right) \eta\left(\mathbf{R}_{\mathrm{tr}}+\mathbf{e}_{\boldsymbol{\mu}}\right) P\left(\mathbf{R}_{\mathrm{tr}}, \eta ; t\right) \\
\approx & \left(\sum_{\mathbf{R}_{\mathrm{tr}}, \eta} \eta\left(\mathbf{R}_{\mathbf{t r}}+\boldsymbol{\lambda}\right) P\left(\mathbf{R}_{\mathrm{tr}}, \eta ; t\right)\right)\left(\sum_{\mathbf{R}_{\mathrm{tr}}, \eta} \eta\left(\mathbf{R}_{\mathrm{tr}}+\mathbf{e}_{\boldsymbol{\mu}}\right) P\left(\mathbf{R}_{\mathbf{t r}}, \eta ; t\right)\right) \\
= & k(\boldsymbol{\lambda} ; t) k\left(\mathbf{e}_{\boldsymbol{\mu}} ; t\right)
\end{aligned}
$$

Some arguments justifying such an approximation can be found in Ref.[40].

Using the approximation in Eq.(13), we obtain

$$
2 d \tau^{*} \partial_{t} k(\boldsymbol{\lambda} ; t)=\tilde{L} k(\boldsymbol{\lambda} ; t)+2 d f
$$

which holds for all $\boldsymbol{\lambda}$, except for $\boldsymbol{\lambda}=\left\{\mathbf{0}, \pm \mathbf{e}_{\mathbf{1}}, \mathbf{e}_{2} \ldots, \mathbf{e}_{\mathbf{d}}\right\}$. One the other hand, for these special sites $\boldsymbol{\lambda}=\mathbf{e}_{\nu}$ with $\nu=\{ \pm 1,2, \ldots, d\}$ we find

$$
2 d \tau^{*} \partial_{t} k\left(\mathbf{e}_{\boldsymbol{\nu}} ; t\right)=\left(\tilde{L}+A_{\nu}\right) k\left(\mathbf{e}_{\boldsymbol{\nu}} ; t\right)+4 f
$$

where $\tilde{L}$ is the operator

$$
\tilde{L} \equiv \sum_{\mu} A_{\mu} \nabla_{\mu}-4(f+g)
$$

and the coefficients $A_{\mu}$ are defined by

$$
A_{\mu}(t) \equiv 1+\frac{2 d \tau^{*}}{\tau} p_{\mu}\left(1-k\left(\mathbf{e}_{\boldsymbol{\mu}} ; t\right)\right)
$$

Note that Eq.(15) represents, from the mathematical point of view, the boundary conditions for the general evolution equation (14), imposed on the sites in the immediate vicinity of the TP. Equations (14) and (15) together with Eq.(9) thus constitute a closed system of equations which suffice computation of all properties of interest, i.e. the time-dependent tracer particle velocity and the particle density profiles as seen from the tracer particle. The general approach to solution of coupled non-linear Eqs.(9),(14) and (15) has been discussed in detail in Ref.[56]. Here we merely note that despite the fact that using the 
decoupling scheme in Eq.(13) we effectively close the system of equations on the level of the pair correlations, solution of Eqs.(14) and (15) still poses serious technical difficulties: Namely, these equations are non-linear with respect to the TP velocity, which enters the gradient term on the rhs of the evolution equations for the pair correlation, and does depend itself on the values of the monolayer particles densities in the immediate vicinity of the TP. Solution of this system of non-linear equations for several particular case will be presented in the next sections.

\section{Biased tracer diffusion in a 1D lattice gas.}

Consider first biased tracer diffusion in the simplest case of a one-dimensional hard-core lattice gas with conserved particles number; that is, a lattice gas in which all particles were initially introduced onto the lattice and particle exchanges with the vapor phase are forbidden $(f=g=0)$. Hence, the particle density $\rho_{s}$ on the lattice is kept constant all the time. For simplicity, we also set here the spacing $\sigma=1$ and $\tau=\tau^{*}=1$. These parameters can be trivially restored in final results.

In absence of external field acting on the $\mathrm{TP}$, when its transition probabilities are symmetric, tracer dynamics has been studied first in Refs.[18-20] and the TP mean-square displacement $<X^{2}(t)>$ has been determined exactly:

$$
<X^{2}(t)>=\frac{\left(1-\rho_{s}\right)}{\rho_{s}} \sqrt{\frac{2 t}{\pi}},
$$

which thus follows an anomalous diffusion law in Eq.(2) with $\alpha=1 / 2$. Consequently, the "diffusivity" $\mathrm{D}(\mathrm{t})$ is given here by

$$
D(t)=\frac{\left(1-\rho_{s}\right)}{\rho_{s}} \frac{1}{\sqrt{2 \pi t}}
$$

and vanishes as $t \rightarrow \infty$.

Now, if we charge the TP and switch on an external electric field, how will the TP velocity and the mobility behave? An early incomplete answer was presented in Ref.[57] which focused on the extreme case of an infinitely strong electric field $E \rightarrow+\infty$ such that 
the TP performs a totally directed random walk constrained by hard-core particles. By noticing that such a model is tantamount to the one-dimensional Stefan freezing problem or the problem of directional solidification [58], (the role of the latent heat being played by the lattice gas particles), it was found that in this extreme case the TP mean velocity follows

$$
V(t)=\frac{\gamma_{\infty}}{\sqrt{t}}
$$

where $\gamma_{\infty}$ is a function of $\rho_{s}$ defined implicitly by

$$
\sqrt{\frac{\pi}{2}} \gamma_{\infty} \exp \left(\frac{\gamma_{\infty}^{2}}{2}\right) \operatorname{erfc}\left(\frac{\gamma_{\infty}}{\sqrt{2}}\right)=1-\rho_{s}
$$

$\operatorname{erfc}(\mathrm{x})$ being the complementary error function. Hence, the TP mean velocity in the biased case decays at the exactly the same rate as the TP "diffusivity" in the unbiased case! In view of such a coincidence in the behavior, one is, of course, prompted to consider the general case $E<\infty$ and check the prefactors in Eq.(20) having in mind to verify the validity of Eq.(5) for anomalous tracer diffusion in such random dynamical environments [59].

The general case of arbitrary $0<E \leq \infty$ has been studied in Ref.[41] within the framework of the approach outlined in Section 2 and subsequently, in terms of a rigorous probabilistic approach in Ref.[42]. Results of these two approaches coincide, which justifies somehow the decoupling approximation in Eq.(13). It has been found that in this general case the TP mean velocity $V(t)$ obeys at sufficiently large times the form of Eq.(20), i.e. $V(t)=\gamma_{E} / \sqrt{t}$, in which law the prefactor $\gamma_{E}$ is determined by the following transcedental equation $[41,42]$ :

$$
\begin{aligned}
& \left(I_{+}\left(\gamma_{E}\right)-1+\frac{\rho_{s}}{1-\exp (-\beta E)}\right) \times \\
\times \quad & \left(I_{-}\left(\gamma_{E}\right)+1+\frac{\rho_{s}}{\exp (\beta E)-1}\right)=\frac{\exp (\beta E) \rho_{s}^{2}}{(\exp (\beta E)-1)^{2}},
\end{aligned}
$$

where

$$
I_{ \pm}\left(\gamma_{E}\right)=\sqrt{\frac{\pi}{2}} \gamma_{E} \exp \left(\frac{\gamma_{E}^{2}}{2}\right)\left[1 \mp \operatorname{erf}\left(\frac{\gamma_{E}}{\sqrt{2}}\right)\right]
$$


In the limit $\beta E \ll 1$ the solution of the transcedental Eq.(22) has the form

$$
\gamma_{E} \approx \frac{\left(1-\rho_{s}\right)}{\rho_{s}} \sqrt{\frac{2}{\pi}} \frac{\beta E}{2}
$$

which implies that the time-dependent mobility in this case is given by

$$
\mu(t)=\frac{\left(1-\rho_{s}\right)}{\rho_{s}} \frac{\beta}{\sqrt{2 \pi t}} .
$$

Finally, by comparing the prefactors in the latter equation and Eq.(19), we find that the generalized Einstein relation in Eq.(5) holds exactly for such an anomalous diffusion!

To close this section, we make several comments on the particle density distribution as seen from the tracer particle. Since the TP has a non-zero mean displacement, while the lattice gas particles have symmetric hopping probabilities and hence, a zero mean displacement, they tend to accumulate in front of the TP. Consequently, a condensed, traffic jam-like region is formed in front of the TP, and a depleted by the lattice-gas particles region past the TP emerges. As a matter of fact, such a inhomogeneous density distribution is not stationary: the size of the traffic jam-like region, in which the particle density profile $k(\lambda, t)$ is nearly constant and larger than $\rho_{s}, k(\lambda, t)=\rho_{s} /\left(1+I_{+}\left(\gamma_{E}\right)\right)$, grows in proportion to the TP mean displacement. In a similar fashion, the size of the depleted region with a constant density $k(\lambda, t)=\rho_{s} /\left(1+I_{-}\left(\gamma_{E}\right)\right)<\rho_{s}$ also grows in proportion to $<X(t)>=V(t) t$.

\section{4 "Shock" propagation in a non-equilibrium 1D lat- tice gas.}

In this section we address the question of the validity of the generalized Einstein relation in a somewhat exotic situation in which the initial particle distributions from both sides of the TP has an $S$-shape, shock-like profile characterized by two different mean densities [43]. That is, as depicted in Fig.2, we suppose that the TP is initially at the origin, and the particles mean densities from the left and from the right of the origin, which we denote 
as $\rho_{-}$and $\rho_{+}$, respectively, are not equal to each other. The TP thus defines position of the "shock front". Without of lack of generality, we suppose that $\rho_{-} \geq \rho_{+} \geq 0$, and will call in what follows the particle phase which initially occupied the left half-line as the high-density phase (HDP), while the phase initially occupying the right half-line will be referred to as the low-density phase (LDP).

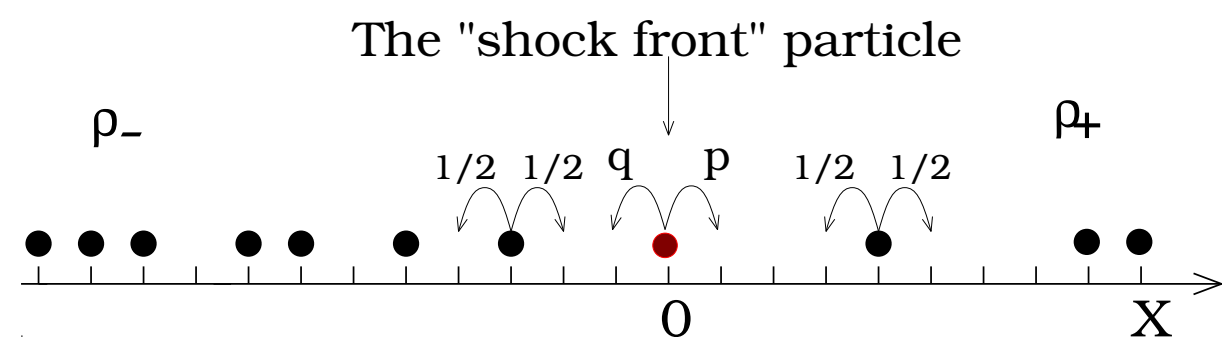

Figure 2: Initial "shock" configuration of the lattice gas particles on a one-dimensional lattice. $\rho_{-}$is the initial mean density of particles at the half-line $-\infty<X<0$ and $\rho_{+}$is the mean density of particles at the half-line $0<X<\infty$. All lattice gas particles, excluding the TP (the "shock" front particle) have symmetric transition probabilities. The TP has asymmetric transition probabilities and experiences the bias in the negative direction.

Further on, after deposition on the lattice, particles start to perform symmetric, (i.e. with equal probabilities for going to the left or to the right), hopping motion between the nearest lattice sites under the constraint that neither two particles can simultaneously occupy the same lattice site and can not pass through each other. Next, we stipulate that the TP is subject to a constant force $F$ which favors its jumps in a preferential direction. Thus for the TP the probabilities of going to the right $(p)$ and to the left $(q)$ will be different from each other. In most situations we will suppose that $F$ is directed towards the HDP; we adopt the convention that in this case $F$ is negative, $F \leq 0$; the TP hopping probabilities $p$ and $q$ are related to the force and the reciprocal temperature $\beta$ through $p / q=\exp (\beta F)$ and $p+q=1$. From the physical point of view, such a constant force can be understood as an effective boundary tension derived from the solid-on-solid-model Hamiltonian of the phaseseparating boundary [60-62] and mimic, in a mean-field fashion, the presence of attractive 
interactions between the lattice-gas particles which are not explicitly included into the model. Relation between $p, q$ and the amplitude of attractive interparticle interactions has been discussed in Refs.[63] and [64].

The TP dynamics in such a system has been analysed in Refs. [43] and [42] and it has been found that the TP mean velocity obeys $\langle V(t)\rangle=\gamma_{F} / \sqrt{t}$ at sufficiently large times, in which equation the prefactor $\gamma_{F}$ is determined implicitly as the solution of the following equation:

$$
\frac{q \rho_{-}}{1+I_{-}\left(\gamma_{F}\right)}-\frac{p \rho_{+}}{1-I_{+}\left(\gamma_{F}\right)}=q-p
$$

which generalizes the result of the previous section for the case of initially unequal mean densities.

Equation (26) predicts that three different regimes can take place depending on the relation between $p / q$ and $\rho_{ \pm}$:

(1) When $p\left(1-\rho_{+}\right)>q\left(1-\rho_{-}\right)$the parameter $\gamma_{F}$ is finite and positive, which means that the HDP expands compressing the LDP. In the particular case $p / q=1$ and $\rho_{+}=0$ the parameter $\gamma_{F}$ appears to be a positive, logarithmically growing with time function, which behavior agrees with the results of Ref.[66].

(2) When $p\left(1-\rho_{+}\right)<q\left(1-\rho_{-}\right)$the parameter $\gamma_{F}$ is less than zero - the expanding LDP and the applied force effectively compress the HDP.

(3) When $p\left(1-\rho_{+}\right)=q\left(1-\rho_{-}\right)$, the parameter $\gamma_{F} \equiv 0$. This relation between the system parameters when the HDP and the LDP are in equilibrium with each other and the TP mean displacement is zero, was found also in Ref.[39] from the analysis of the stationary behavior in a finite one-dimensional lattice gas.

"Shock front", or, in other words, the TP propagation in cases (1) and (2) has been discussed in detail in Ref.[41]. Here, in view of our interest in the Einstein relation, we will focus on the case (3) in which situation the high- and low-density phases coexist such that the TP mean displacement is equal to zero. On the other hand, it is clear that also in this case the TP will wander randomly around the equilibrium position and one may expect that its mean-square displacement will not be equal to zero. 
To determine the TP mean-square displacement $\left\langle X^{2}(t)>\right.$ we may try to extend the approach outlined in Section 2 which will require some more cumbersome analysis. We will however pursue a different, heuristic approach and instead of evaluating $\left\langle X^{2}(t)\right\rangle$ in terms of the approach of Section 2 we will resort to the Einstein relation in Eq.(5) assuming that it also holds in the inhomogeneous situation under study. Our result will be then checked by numeric simulations.

To do this, we note first that contrary to the model of Section 3, here the TP does not move, on average, when the external force $F$ acting on this particle is not equal to zero, but rather to some critical value $F_{c}$, such that

$$
F_{c}=\beta^{-1} \ln \left(\frac{1-\rho_{-}}{1-\rho_{+}}\right) .
$$

Hence, it seems reasonable to define the TP mobility in this inhomogeneous case as

$$
\mu(t)=\lim _{F \rightarrow F_{c}} \frac{V(t)}{\left(F-F_{c}\right)}=\frac{1}{\sqrt{t}} \lim _{F \rightarrow F_{c}} \frac{\gamma_{F}}{\left(F-F_{c}\right)}
$$

Further on, we find from Eq.(26) that in the limit $\beta\left(F-F_{c}\right) \ll 1$ the prefactor $\gamma_{F}$ follows

$$
\gamma_{F} \sim \sqrt{\frac{2}{\pi}} \frac{p\left(1-\rho_{+}\right)-q\left(1-\rho_{-}\right)}{q \rho_{-}+p \rho_{+}},
$$

which implies that $\mu(t)$ obeys

$$
\mu(t)=\beta \frac{\left(1-\rho_{-}\right)\left(1-\rho_{+}\right)}{\left(\rho_{-}+\rho_{+}-2 \rho_{-} \rho_{+}\right)} \sqrt{\frac{2}{\pi t}} .
$$

Now, assuming that Eq.(5) holds, we get

$$
<X^{2}(t)>=\frac{\left(1-\rho_{-}\right)\left(1-\rho_{+}\right)}{\left(\rho_{-}+\rho_{+}-2 \rho_{-} \rho_{+}\right)} \sqrt{\frac{8 t}{\pi}},
$$

which reduces to the classical result in Eq.(18) in the limit $\rho_{-}=\rho_{+}$.

In Fig.3 we present results of extensive Monte Carlo simulations which confirm the result in Eq.(31) and hence, signify that the Einstein relation seemingly works in this inhomogeneous case. 


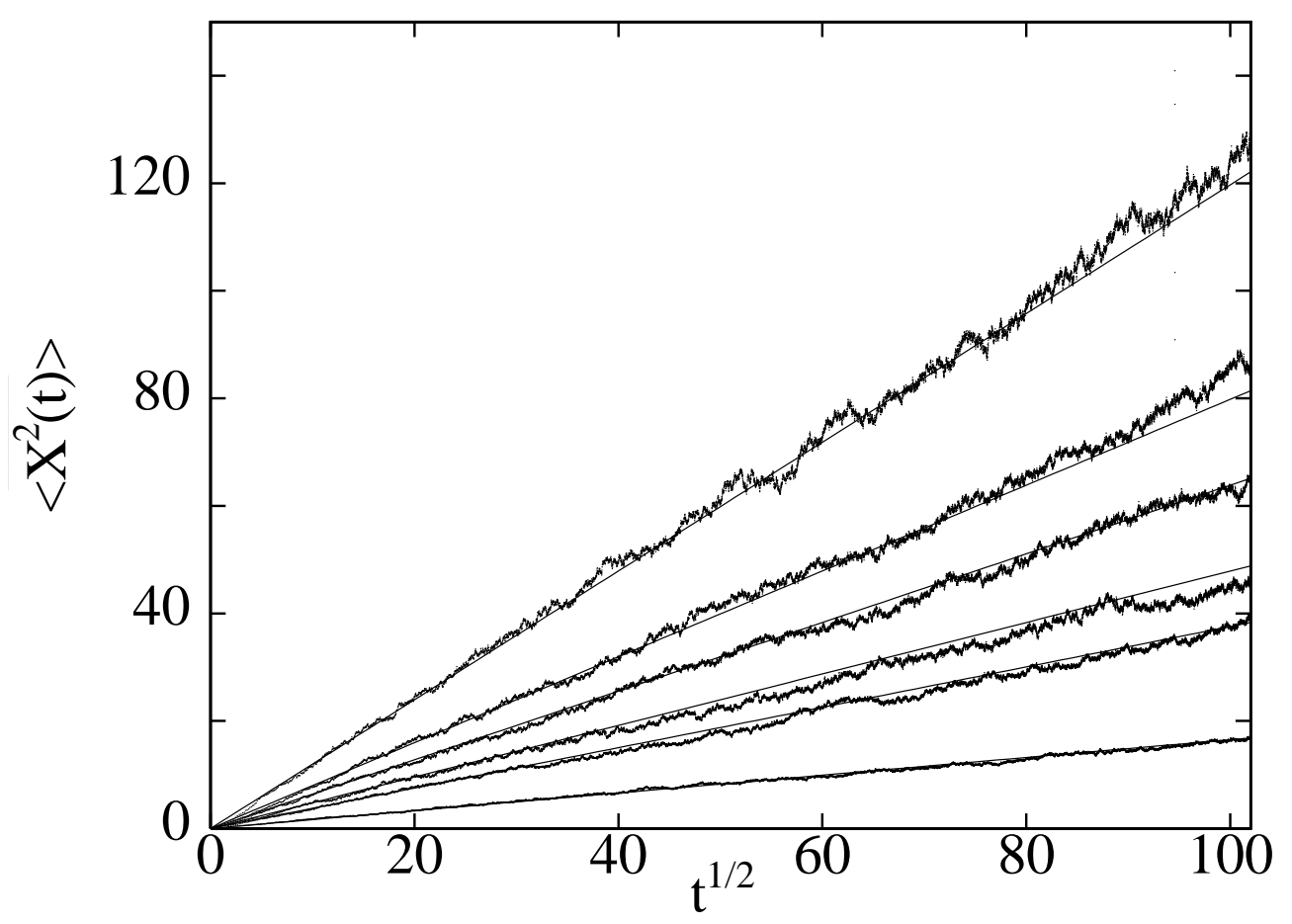

Figure 3: Mean-square displacement of the TP in the critical case $p / q=\left(1-\rho_{-}\right) /\left(1-\rho_{+}\right)$. The solid lines show our analytical prediction from Eq.(31) and the noisy lines present the results of Monte Carlo simulations. The curves from top to bottom correspond to the following values of the parameters $\left(\rho_{-}, \rho_{+}\right)$: The first two curves are the analytical result in Eq.(18) and numerical data for the symmetric cases $(0.4,0.4)$ and $(0.5,0.5)$. The lower curves correspond to $(0.6,0.5)$, $(0.9,0.4),(0.7,0.5)$ and $(0.8,0.2)$ respectively.

\section{Biased TP diffusion in a 1D lattice gas in contact with a reservoir.}

We turn now to discussion of the TP biased diffusion in situation in which the reservoir is present and particle exchanges with the reservoir are allowed; that is, when both the parameters $f$ and $g$ are not equal to zero.

In one-dimensional systems with continuous particles exchanges with a reservoir, which situation is appropriate to adsorption on polymer chains $[67,68]$, the biased TP will ulti- 
mately move with a constant velocity $V$ and particles distribution around the TP will be characterized by stationary density profiles $k(\lambda)[40]$. Here, the general solution of Eqs.(14) and (15) has the following form:

$$
k(\lambda)=\rho_{s}+K_{ \pm} \exp \left(-\sigma|n| / \lambda_{ \pm}\right), \quad \lambda=\sigma n, \quad n \in Z
$$

where the characteristic lengths $\lambda_{ \pm}$obey

$$
\lambda_{ \pm}=\mp \sigma \ln ^{-1}\left[\frac{A_{1}+A_{-1}+2(f+g) \mp \sqrt{\left(A_{1}+A_{-1}+2(f+g)\right)^{2}-4 A_{1} A_{-1}}}{2 A_{1}}\right],
$$

while the amplitudes $K_{ \pm}$are given respectively by

$$
K_{+}=\rho_{s} \frac{A_{1}-A_{-1}}{A_{-1}-A_{1} \exp \left(-\sigma / \lambda_{+}\right)}
$$

and

$$
K_{-}=\rho_{s} \frac{A_{1}-A_{-1}}{A_{-1} \exp \left(-\sigma / \lambda_{-}\right)-A_{1}}
$$

Note that $\lambda_{-}>\lambda_{+}$, and consequently, the local density past the TP approaches its nonperturbed value $\rho_{s}$ slower than in front of it; this signifies that correlations between the TP position and particle distribution are stronger past the TP. Next, $K_{+}$is always positive, while $K_{-}<0$; this means that the density profile is a non-monotoneous function of $\lambda$ and is characterized by a jammed region in front of the TP, in which the local density is higher than $\rho_{s}$, and a depleted region past the TP in which the density is lower than $\rho_{s}$. This is, of course, quite similar to the behavior observed in situations in which exchanges with the reservoir are not allowed (Sections 3 and 4); essential difference is, however, that here the inhomogeneous particles distribution around the TP attains a stationary form.

Now, we are in position to obtain a system of two closed-form non-linear equations determining implicitly the unknown parameters $A_{1}$ and $A_{-1}$, which will allow us to compute the TP terminal velocity, related to $A_{ \pm 1}$ through $V=\sigma\left(A_{1}-A_{-1}\right) / 2 \tau^{*}$. Substituting Eq.(32) into Eq.(17), we find

$$
A_{1}=1+\frac{p_{1} \tau^{*}}{\tau}\left[1-\rho_{s}-\rho_{s} \frac{A_{1}-A_{-1}}{A_{-1} \exp \left(\sigma / \lambda_{+}^{\prime}\right)-A_{1}}\right]
$$




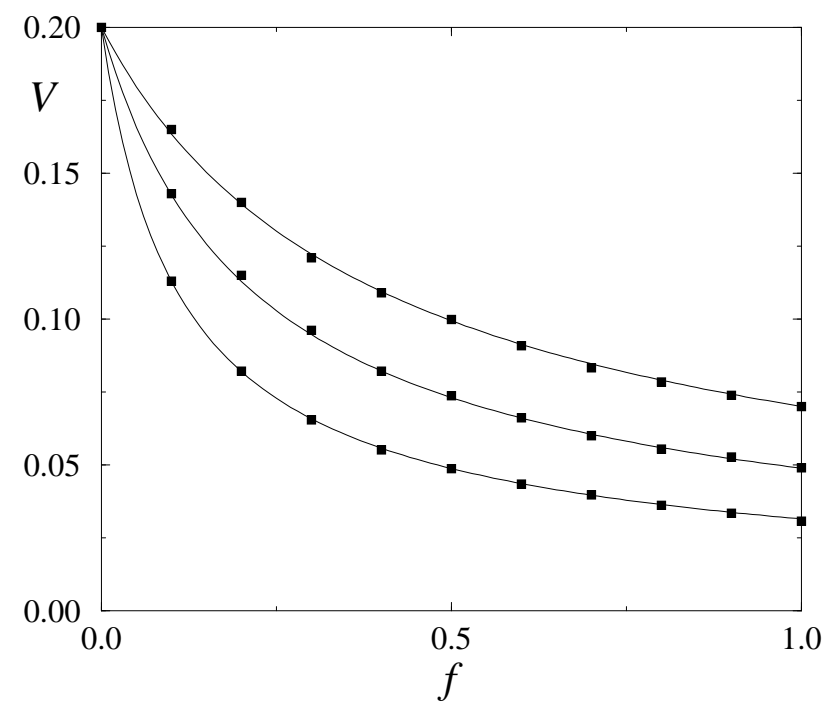

Figure 4: Terminal velocity of the TP as a function of the "creation" probability $f$ at different values of the parameter $g$. The TP hopping probabilities are $p_{+1}=0.6$ and $p_{-1}=0.4$. The solid lines give the analytical solution while the filled squares denote the results of Monte-Carlo simulations. Upper curves correspond to $g=0.8$, the intermediate - to $g=0.5$ and the lower to $g=0.3$, respectively.

and

$$
A_{-1}=1+\frac{p_{-1} \tau^{*}}{\tau}\left[1-\rho_{s}-\rho_{s} \frac{A_{1}-A_{-1}}{A_{-1}-A_{1} \exp \left(\sigma / \lambda_{-}^{\prime}\right)}\right]
$$

Resolution of this system leads then to the stationary velocity of the TP (see Fig.4) as well as the density profiles (see Fig.5). For arbitrary values of $p, f$ and $g$ the parameters $A_{ \pm 1}$, defined by Eqs.(36) and (37), and consequently, the terminal velocity $V$ can be determined only numerically (see Figs.4 to 6). However, $V$ can be found analytically in the explicit form in the limit of a vanishingly small force $E, \beta E \ll 1$. Expanding $A_{ \pm 1}$ in the Taylor series in powers of $E$ and retaining only linear with $E$ terms, we find that the TP velocity follows

$$
V \sim \zeta^{-1} E
$$

which relation can be thought off as the analog of the Stokes formula for driven motion in a one-dimensional lattice gas undergoing continuous particles exchanges with the vapor 
phase. Equation (38) signifies that the frictional force exerted on the TP by the lattice gas particles is viscous. The friction coefficient, i.e. the proportionality factor in Eq.(38) is given explicitly by

$$
\zeta=\frac{2 \tau}{\beta \sigma^{2}\left(1-\rho_{s}\right)}\left[1+\frac{\rho_{s} \tau^{*}}{\tau(f+g)} \frac{2}{1+\sqrt{1+2\left(1+\tau^{*}\left(1-\rho_{s}\right) / \tau\right) /(f+g)}}\right]
$$

Note that the friction coefficient in Eq.(39) can be written down as the sum of two contributions $\zeta=\zeta_{c m}+\zeta_{c o o p}$. The first one, $\zeta_{c m}=2 \tau / \beta \sigma^{2}\left(1-\rho_{s}\right)$ is a typical mean-field result and corresponds to a perfertly homogeneous lattice gas (see discussion following Eq.(10)). The second one,

$$
\zeta_{\text {coop }}=\frac{8 \tau^{*} \rho_{s}}{\beta \sigma^{2}\left(1-\rho_{s}\right)(f+g)} \frac{1}{1+\sqrt{1+2\left(1+\tau^{*}\left(1-\rho_{s}\right) / \tau\right) /(f+g)}},
$$

has, however, a more complicated origin. Namely, it reflects a cooperative behavior emerging in the lattice gas, associated with the formation of inhomogeneous density profiles (see Fig.5) - the formation of a "traffic jam" in front of the TP and a "depleted" region past the TP (for more details, see Ref.[40]). The characteristic lengths of these two regions as well as the amplitudes $K_{ \pm}$depend on the magnitude of the TP velocity; on the other hand, the TP velocity is itself dependent on the density profiles, in virtue of Eq.(9). This results in an intricate interplay between the jamming effect of the $\mathrm{TP}$ and smoothening of the created inhomogeneities by diffusive processes. Note also that cooperative behavior becomes most prominent in the conserved particle number limit [41]. Setting $f, g \rightarrow 0$, while keeping their ratio fixed (which insures that $\rho_{s}$ stays constant), one notices that $\zeta_{\text {coop }}$ gets infinitely large. As a matter of fact, as we have shown already in section 3 , in such a situation no stationary density profiles around the TP exist.

In order to check our analytical predictions, we have performed numerical Monte Carlo simulations. Results of these simulations, performed at different values of the parameters $f, g$, and $p_{1}$, are also represented in Figs. 4 and 5 .

Consider finally the situation with $E=0$, in which case the terminal velocity vanishes and one for which one expects conventional diffusive motion with the mean square displacement of the form od Eq.(1). Heuristically, we can evaluate $D$ for the system under 


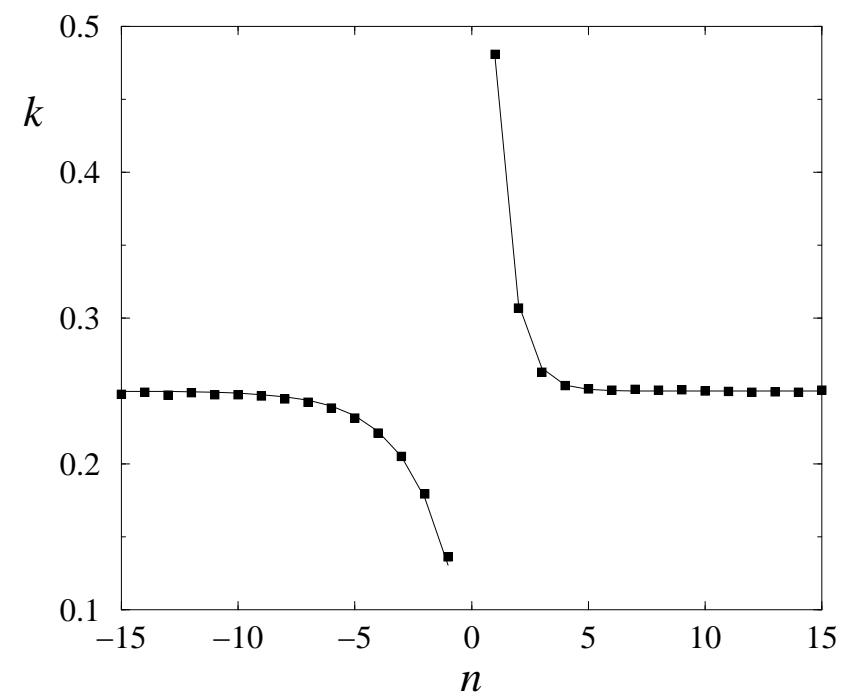

Figure 5: Density profile around stationary moving TP for $f=0.1, g=0.3$ and $p=0.98$. The solid line is the plot of the analytical solution. Filled squares are the results of Monte-Carlo simulations.

study if we assume the validity of the Einstein relation in Eq.(5). Noticing that here the biased TP mobility $\mu$ is just $\mu=1 / \zeta$, we find [40]:

$$
D=\frac{\sigma^{2}\left(1-\rho_{s}\right)}{2 \tau}\left\{1+\frac{\rho_{s} \tau^{*}}{\tau(f+g)} \frac{2}{1+\sqrt{1+2\left(1+\tau^{*}\left(1-\rho_{s}\right) / \tau\right) /(f+g)}}\right\}^{-1} .
$$

Monte Carlo simulations (see Fig.6) of the system evidently confirm our prediction for $D$ given by Eq.(41), and hence, confirm the validity of the Einstein relation for the system under study. This is, of course, not an unexpected, but still a non-trivial result [39].

\section{Biased TP diffusion in 2D and 3D lattice gases.}

We turn now to the analysis of a biased TP diffusion in higher dimensional (2D and 3D) lattice gases exposed to a vapor phase. Here, the situation gets somewhat more difficult from the computational point of view; we have now to solve the partial difference equations problem (14), (15) rather than the mere difference equations arising in the one-dimensional 


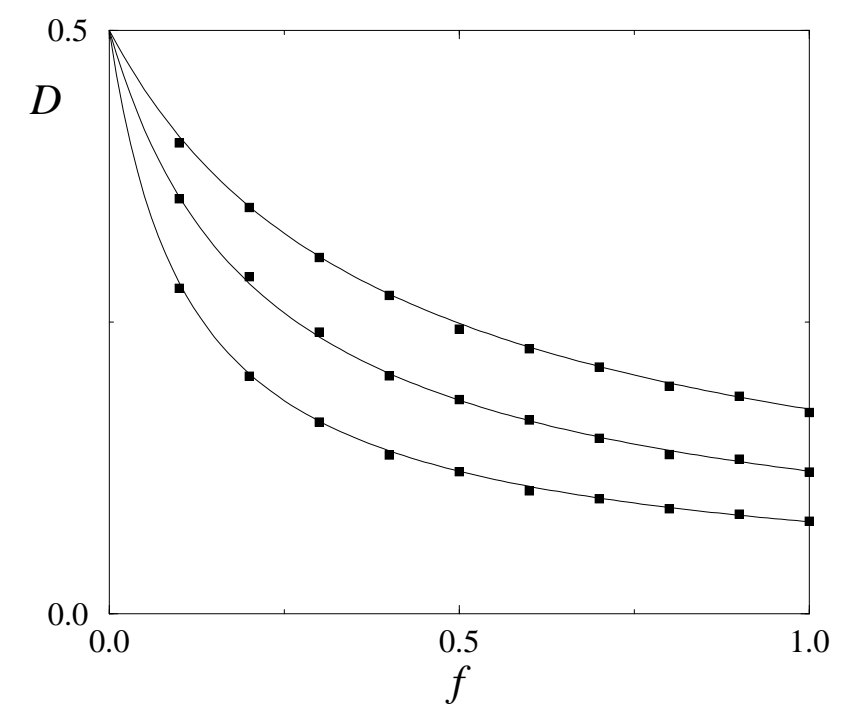

Figure 6: Self-diffusion coefficient of the probe molecule as a function of the adsorption probability $f$. Notations and values of $g$ are the same as in Figs.4.

case.

Below we briefly outline such a solution for a two-dimensional case. Results for threedimensions are obtained along essentially the same lines and here we display them without derivation. Solution in two-dimensions can be found in a most convenient fashion if we introduce the generating function for the particle density profiles, defined as

$$
H\left(w_{1}, w_{2}\right) \equiv \sum_{n_{1}=-\infty}^{+\infty} \sum_{n_{2}=-\infty}^{+\infty}\left(\rho_{s}-k\left(n_{1}, n_{2}\right)\right) w_{1}^{n_{1}} w_{2}^{n_{2}}
$$

Multiplying both sides of Eqs. (14) and (15) by $w_{1}^{n_{1}} w_{2}^{n_{2}}$, and performing summations over $n_{1}$ and $n_{2}$, we find that $H\left(w_{1}, w_{2}\right)$ is given explicitly by

$$
H\left(w_{1}, w_{2}\right)=-K\left(w_{1}, w_{2}\right)\left\{A_{1} w_{1}^{-1}+A_{-1} w_{1}+A_{2}\left(w_{2}+w_{2}^{-1}\right)-\alpha\right\}^{-1}
$$

where $\alpha \equiv \sum_{\nu} A_{\nu}+4(f+g)$ and

$$
K\left(w_{1}, w_{2}\right) \equiv \sum_{\nu} A_{\nu}\left(w_{|\nu|}^{\nu /|\nu|}-1\right)\left(k\left(\mathbf{e}_{\boldsymbol{\nu}}\right)-\rho_{s}\right)+\rho_{s}\left(A_{1}-A_{-1}\right)\left(w_{1}-w_{1}^{-1}\right)
$$

Equations (43) and (44) determine the generation function for the density profiles exactly. 
Before we proceed to the inversion of $H\left(w_{1}, w_{2}\right)$ with respect to the variables $w_{1}$ and $w_{2}$, we note that we can access interesting integral characteristic of the density profiles directly using the result in Eqs.(43) and (44). Namely, as we have already remarked, the presence of the driven TP induces an inhomogeneous density distribution in the lattice. One can thus pose a natural question whether equilibrium between adsorption and desorption processes gets shifted due to such a perturbancy, i.e. whether the equilibrium density in the lattice gas is different from that given by Eq.(6). The answer is trivially "no" in the case when the particles number is explicitly conserved, but in the general case with arbitrary $f$ and $g$ this is not at all evident: similarly to the behavior in one-dimensional system one expects that also in two-dimensions the density profiles are asymmetric as seen from the stationary moving TP and are characterized by a condensed, "traffic-jam"-like region in front of and a depleted region past the TP. One anticipates then that the desorption events are favored in front of the TP, while the adsorption events are evidently suppressed by the excess density. On the other hand, past the TP desorption is diminished due to the particles depletion while adsorption may proceed more readily due to the same reason. It is thus not at all clear a priori whether these two effects can compensate each other exactly, in view of a possible asymmetry of the density profiles, as it happens in the one-dimensional model (see Fig.5).

For this purpose, we study the behavior of the integral deviation $\Omega$ of the density from the equilibrium value $\rho_{s}$, i.e. $\Omega \equiv \sum_{n_{1}=-\infty}^{+\infty} \sum_{n_{2}=-\infty}^{+\infty}\left(\rho_{s}-k\left(n_{1}, n_{2}\right)\right)$, which can be computed straightforwardly from Eqs.(43) and (44) by setting both $w_{1}$ and $w_{2}$ equal to unity. Noticing that $K\left(w_{1}=1, w_{2}=1\right)=0$, and that $A_{1}+A_{-1}+2 A_{2}-\alpha=-4(f+g)$, i.e. is strictly negative as soon as adsorption/desorption processes are present, we obtain then that $\Omega$ is strictly equal to 0 . This implies, in turn, that the perturbancy of the density distribution in the lattice gas created by the driven TP does not shift the global balance between the adsorption and desorption events.

Inversion of the generating function with respect to $w_{1}$ and $w_{2}$ requires quite an involved mathematical analysis, which has been presented in detail in Ref.[56]. General solution for 
the density profiles reads:

$$
\begin{aligned}
k\left(n_{1}, n_{2}\right)=\rho_{s} & +\alpha^{-1}\left\{\sum _ { \nu } A _ { \nu } \left(k\left(\mathbf{e}_{\boldsymbol{\nu}}\right)-\right.\right. \\
& \left.\left.-\rho_{s}\right) \nabla_{-\nu} F_{n_{1}, n_{2}}-\rho_{s}\left(A_{1}-A_{-1}\right)\left(\nabla_{1}-\nabla_{-1}\right) F_{n_{1}, n_{2}}\right\} .
\end{aligned}
$$

with

$$
F_{n_{1}, n_{2}}=\left(\frac{A_{-1}}{A_{1}}\right)^{n_{1} / 2} \int_{0}^{\infty} e^{-t} \mathrm{I}_{n_{1}}\left(2 \alpha^{-1} \sqrt{A_{1} A_{-1}} t\right) \mathrm{I}_{n_{2}}\left(2 \alpha^{-1} A_{2} t\right) \mathrm{d} t
$$

where $\mathrm{I}_{n}(z)$ stands for the modified Bessel function. We mention that $F_{n_{1}, n_{2}}$ has an interesting physical interpretation in terms of the generating function of a random walk of a single particle (that is, in absence of the particles environment) [69].

Now, the Eqs.(45) and (46) display $k\left(n_{1}, n_{2}\right)$ as a function of the coefficients $A_{\nu}$ that remain to be determined. As a matter of fact, these coefficients depend themselves on the local densities in the immediate vicinity of the tracer, i.e. on $k\left(\mathbf{e}_{\nu}\right)$. This implies that we have to determine them from Eqs.(45) and (46) in a self-consistent way [56]. Some analysis (see Ref.[56]) shows that $A_{\nu}$ are determined implicitly as the solution of the following system of three non-linear matrix equations

$$
\forall \nu=\{ \pm 1,2\}, \quad A_{\nu}=1+\frac{4 \tau^{*}}{\tau} p_{\nu}\left\{1-\rho_{s}-\rho_{s}\left(A_{1}-A_{-1}\right) \frac{\operatorname{det} \tilde{C}_{\nu}}{\operatorname{det} \tilde{C}}\right\}
$$

where

$$
\tilde{C}=\left(\begin{array}{ccc}
A_{1} \nabla_{-1} F_{\mathbf{e}_{1}}-\alpha & A_{-1} \nabla_{1} F_{\mathbf{e}_{1}} & A_{2} \nabla_{-2} F_{\mathbf{e}_{1}} \\
A_{1} \nabla_{-1} F_{\mathbf{e}_{-1}} & A_{-1} \nabla_{1} F_{\mathbf{e}_{-1}}-\alpha & A_{2} \nabla_{-2} F_{\mathbf{e}_{-1}} \\
A_{1} \nabla_{-1} F_{\mathbf{e}_{2}} & A_{-1} \nabla_{1} F_{\mathbf{e}_{2}} & A_{2} \nabla_{-2} F_{\mathbf{e}_{2}}-\alpha
\end{array}\right)
$$

the matrix $\tilde{C}_{\nu}$ stands for the matrix obtained from $\tilde{C}$ by replacing the $\nu$-th column by the column-vector $\tilde{F}$,

$$
\tilde{F}=\left(\begin{array}{c}
\left(\nabla_{1}-\nabla_{-1}\right) F_{\mathbf{e}_{1}} \\
\left(\nabla_{1}-\nabla_{-1}\right) F_{\mathbf{e}_{-1}} \\
\left(\nabla_{1}-\nabla_{-1}\right) F_{\mathbf{e}_{2}}
\end{array}\right)
$$

while $k\left(\mathbf{e}_{\boldsymbol{\nu}}\right)$ are expressed in terms of $A_{\nu}$ as

$$
k\left(\mathbf{e}_{\boldsymbol{\nu}}\right)=1+\frac{\tau}{4 \tau^{*} p_{\nu}}\left(1-A_{\nu}\right)
$$


Lastly, we find that in 2D the TP terminal velocity obeys

$$
V=\frac{\sigma}{\tau}\left(p_{1}-p_{-1}\right)\left(1-\rho_{s}\right)\left\{1+\rho_{s} \frac{4 \tau^{*}}{\tau} \frac{p_{1} \operatorname{det} \tilde{C}_{1}-p_{-1} \operatorname{det} \tilde{C}_{-1}}{\operatorname{det} \tilde{C}}\right\}^{-1},
$$

which represents the general force-velocity relation for the system under study, valid for arbitrary magnitude of the external bias and arbitrary values of other system's parameters.

We turn now to the limit $\beta E \ll 1$, in which case the problem simplifies considerably and allows to obtain explicit results for the local densities in the immediate vicinity of the TP and consequently, for the TP terminal velocity and its diffusivity.

In this limit, we arrive again at a Stokes-type formula of the form $V \sim E / \zeta$, where now

$$
\zeta=\frac{4 \tau}{\beta \sigma^{2}\left(1-\rho_{s}\right)}\left\{1+\frac{\tau^{*}}{\tau} \frac{\rho_{s}}{\left(f+g+1+\tau^{*}\left(1-\rho_{s}\right) / \tau\right)\left(\mathcal{L}_{2}(x)-x\right)}\right\},
$$

with

$$
x=\frac{1}{2} \frac{1+\tau^{*}\left(1-\rho_{s}\right) / \tau}{f+g+1+\tau^{*}\left(1-\rho_{s}\right) / \tau},
$$

and

$$
\mathcal{L}_{2}(x) \equiv\left\{\int_{0}^{\infty} e^{-t}\left(\left(\mathrm{I}_{0}(x t)-\mathrm{I}_{2}(x t)\right) \mathrm{I}_{0}(x t) \mathrm{d} t\right\}^{-1} .\right.
$$

Note that we again are able to single out two physical meaningful contributions to the friction coefficient $\zeta$. Namely, the first term on the rhs of Eq.(52) is just the mean-fieldtype result corresponding to a perfectly stirred lattice gas, in which correlations between the TP and the lattice gas particles are discarded. The second term, similarly to the one-dimensional case, mirrors the cooperative behavior emerging in the lattice gas and is associated with the backflow effects. In contrast to the one-dimensional case, however, the contribution to the overall friction coefficient stemming out of the cooperative effects remains finite in the conserved particles limit.

Lastly, assuming a priori that the Einstein relation holds for the system under study and noticing that here again the mobility $\mu=1 / \zeta$, we estimate the TP diffusion coefficient as

$$
D=\frac{\sigma^{2}}{4 \tau}\left(1-\rho_{s}\right)\left\{1-\frac{2 \rho_{s} \tau^{*}}{\tau} \frac{1}{4\left(f+g+1+\tau^{*}\left(1-\rho_{s}\right) / \tau\right) \mathcal{L}_{2}(x)-1+\left(3 \rho_{s}-1\right) \tau^{*} / \tau}\right\} .
$$


It seems now interesting to compare our general result in Eq.(55) against the classical result of Nakazato and Kitahara [52], which describes TP diffusion coefficient in a twodimensional lattice gas with conserved particles number. Setting $f$ and $g$ equal to zero, while assuming that their ratio has a fixed value, $f / g=\rho_{s} /\left(1-\rho_{s}\right)$, we have, using the equality:

$$
\mathcal{L}_{2}(1 / 2)=\frac{\pi}{4(\pi-2)}
$$

that the right-hand-side of Eq.(55) attains the form

$$
\hat{D}=\frac{\sigma^{2}}{4 \tau}\left(1-\rho_{s}\right)\left\{1-\frac{2 \rho_{s} \tau^{*}}{\tau} \frac{1-2 / \pi}{1+\left(1-\rho_{s}\right) \tau^{*} / \tau-(1-2 / \pi)\left(1+\left(1-3 \rho_{s}\right) \tau^{*} / \tau\right)}\right\},
$$

which expression coincides exactly with the earlier result obtained in Refs.[52] and [53] within the framework of a different, compared to ours, analytical technique. The result in Eq.(57) is known to be exact in the limits $\rho_{s} \ll 1$ and $\rho_{s} \sim 1$, and serves as a very good approximation for the self-diffusion coefficient in hard-core lattice gases of arbitrary density [51].

In a similar fashion we find that in 3D the TP terminal velocity obeys $V \sim E / \zeta$, which signifies that also in 3D the force exerted on the TP by the lattice gas particles is viscous, and

$$
\zeta=\frac{6 \tau}{\beta \sigma^{2}\left(1-\rho_{s}\right)}+\frac{12 \rho_{s} \tau^{*}}{\beta \sigma^{2} l\left(1-\rho_{s}\right) \cdot\left(\alpha_{0} \mathcal{L}_{3}\left(2 A_{0} / \alpha_{0}\right)-A_{0}\right)}
$$

where

$$
\begin{gathered}
A_{0}=1+\frac{\tau^{*}}{l \tau}\left(1-\rho_{s}\right), \\
\alpha_{0}=6\left(1+\frac{\tau^{*}\left(1-\rho_{s}\right)}{l \tau}+\frac{f+g}{l}\right),
\end{gathered}
$$

while

$$
\begin{aligned}
\mathcal{L}_{3}(x) & \equiv\left\{\int_{0}^{\infty} e^{-t} \mathrm{I}_{0}^{2}(x t)\left(\mathrm{I}_{0}(x t)-\mathrm{I}_{2}(x t)\right) \mathrm{d} t\right\}^{-1} \\
& =\left\{P(\mathbf{0} ; 3 x)-P\left(2 \mathbf{e}_{\mathbf{1}} ; 3 x\right)\right\}^{-1}
\end{aligned}
$$

$P(\mathbf{R} ; \xi)$ being the generating function,

$$
P(\mathbf{R} ; \xi) \equiv \sum_{j=0}^{+\infty} P_{j}(\mathbf{R}) \xi^{j}
$$


of the probability $P_{j}(B F R)$ that a walker starting at the origin and performing a Polya random walk on the sites of a three-dimensional cubic lattice will arrive on the $j$-th step to the site with the lattice vector $\mathbf{R}[69]$.

Assuming the validity of the Einstein relation, we find next that in three-dimensions, the TP diffusion coefficient reads

$$
D=\frac{\sigma^{2}\left(1-\rho_{s}\right)}{6 \tau}\left\{1-\frac{2 \rho_{s} \tau^{*}}{l \tau}\left(\alpha_{0} \mathcal{L}_{3}\left(2 A_{0} / \alpha_{0}\right)-1+\frac{\tau^{*}\left(3 \rho_{s}-1\right)}{l \tau}\right)^{-1}\right\}
$$

In the particular case of conserved particles number, when $f, g \rightarrow 0$ but their ratio $f / g$ is kept fixed, $f / g=\rho_{s} /\left(1-\rho_{s}\right)$, the latter equation reduces to

$$
\hat{D}=\frac{\sigma^{2}\left(1-\rho_{s}\right)}{6 \tau}\left\{1-\frac{2 \rho_{s} \tau^{*}}{l \tau}\left(6 A_{0} \mathcal{L}(1 / 3)-1+\frac{\tau^{*}\left(3 \rho_{s}-1\right)}{l \tau}\right)^{-1}\right\},
$$

which again coincides with the classic result obtained earlier in Refs. [52] and [53] by a different analytical technique.

Asymptotical behavior of the density profiles at large distances from the TP follows from the analysis of the analyticity properties of the complex function $N(z) \equiv \sum_{n=-\infty}^{+\infty} k(n, 0) z^{n}$. It has been shown in Ref.[56] that in front of the TP, $k(n, 0)$ always decays exponentially with the distance:

$$
k(n, 0) \sim \rho_{s}+K_{+} \frac{\exp \left(-n / \lambda_{+}\right)}{n^{1 / 2}},
$$

where the characteristic length $\lambda_{+}$obeys, in two-dimensions:

$$
\lambda_{+}=\ln ^{-1}\left(\frac{1}{A_{-1}}\left\{\frac{\alpha}{2}-A_{2}+\sqrt{\left(\frac{\alpha}{2}-A_{2}\right)^{2}-A_{1} A_{-1}}\right\}\right)
$$

Note that $\lambda_{+}$stays finite for any values of the system parameters.

On contrary, the behavior of the density profiles at large distances past the tracer qualitatively depends on the physical situation studied. In the general case when exchanges with the particles reservoir are allowed, the decay of the density profiles is still exponential with the distance:

$$
k(-n, 0) \sim \rho_{s}+K_{-} \frac{\exp \left(-n / \lambda_{-}\right)}{n^{1 / 2}}
$$


where

$$
\lambda_{-}=-\ln ^{-1}\left(\frac{1}{A_{-1}}\left\{\frac{\alpha}{2}-A_{2}-\sqrt{\left(\frac{\alpha}{2}-A_{2}\right)^{2}-A_{1} A_{-1}}\right\}\right)
$$

Note that in the general case the characteristic lenghts again, similarly to the one-dimensional case, satisfy the inequality $\lambda_{-}>\lambda_{+}$, which means that the correlations between the TP and the lattice particles are always stronger past than in front of the TP.

Such correlations can even become extremely strong in the special case when the particles exchanges with the vapor phase are forbidden, i.e. in the conserved particles number limit. In this case, we have that $\lambda_{-}$becomes infinitely large and, in the limit $n \rightarrow+\infty$, the particle density follows

$$
k(-n, 0)=\rho_{s}-\frac{K_{-}^{\prime}(d=2)}{n^{3 / 2}}\left(1+\frac{3}{8 n}+\mathcal{O}\left(\frac{1}{n^{2}}\right)\right), \quad K_{-}^{\prime}(d=2)>0,
$$

Remarkably enough, in this case the correlations between the TP position and the parti-

cles distribution vanish algebraically slow with the distance! This implies, in turn, that in the conserved particles number case, the diffusive mixing of the lattice gas is not efficient enough to prevent the appearence of the quasi-long-range order and the medium "remembers" the passage of the TP on a long time and space scale, which signifies very strong memory effects. In three dimensions, an analogous result for the asymptotical behavior of the density profiles as seen from the stationary moving TP is:

$$
k(-n, 0,0) \sim \rho_{s}-\frac{K_{-}^{\prime}(d=3) \ln (n)}{n^{2}}, \quad K_{-}^{\prime}(d=3)>0,
$$

where $K_{-}^{\prime}(d=3)$ is a constant [29].

\section{Biased, single vacancy-mediated TP diffusion.}

We turn finally to the extreme case of very dense lattice gas in which all sites of except one (called in what follows "a vacancy") are filled with identical neutral, hard-core particles (see, Fig.7). The particles move randomly by exchanging their positions with this single vacancy, subject to the hard-core exclusion constraint. The just described model, which represents, in fact, one of the simplest cases of the so-called "slaved diffusion processes", 
has been studied over the years in various guises, such as, for instance, the "constrained dynamics" model of Palmer [71].

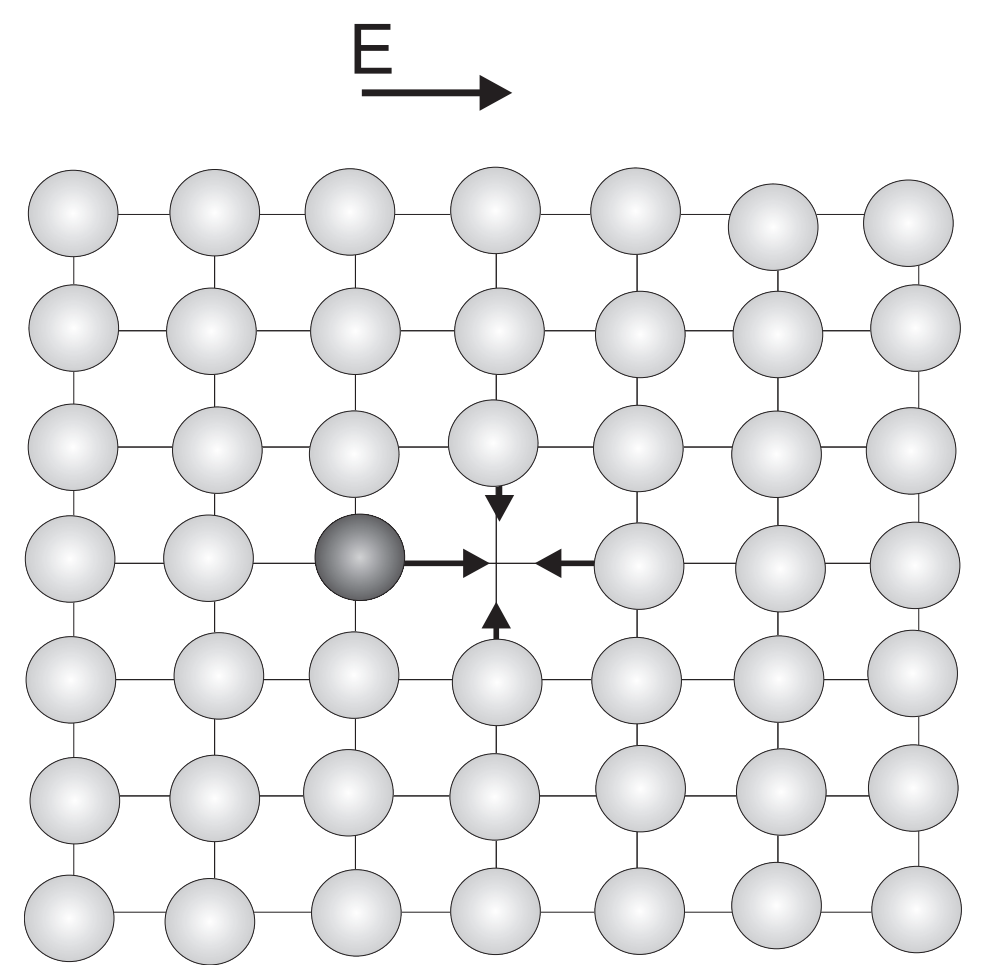

Figure 7: Two-dimensional, infinite in both directions, square lattice in which all sites except one are filled with identical hard-core particles (gray spheres). The black sphere denotes a single tracer particle, which is subject to external field $\mathbf{E}$, oriented in the positive $x_{1}$ direction, and thus has asymmetric hopping probabilities. The arrows of different size depict schematically the hopping probabilities; a larger arrow near the TP indicates that it has a preference for moving in the direction of the applied field.

Brummelhuis and Hilhorst [55] were first to present an exact solution of this model in the lattice formulation. It has been shown that in the presence of a single vacancy the TP trajectories are remarkably confined; the mean-square displacement shows an unbounded growth, but it grows only logarithmically with time,

$$
<\mathbf{R}_{n}^{2}>\sim \frac{\ln (n)}{\pi(\pi-1)}, \quad \text { as } n \rightarrow \infty,
$$


which implies that the TP diffusivity $D_{n}$ obeys

$$
D_{n} \sim \frac{\ln (n)}{4 \pi(\pi-1) n},
$$

and vanishes as time $n$ progresses. Brummelhuis and Hilhorst have also found [55] that at sufficiently large times the probability $P_{n}\left(\mathbf{R}_{n}\right)$ of finding the TP at time moment $n$ at position $\mathbf{R}_{n}$ converges to a limiting form as a function of the scaling variable $\eta=\left|\mathbf{R}_{n}\right| / \sqrt{\ln (n)}$. Still striking, this limiting distribution is not a Gaussian but a modified Bessel function $K_{0}(\eta)$, which signifies that the successive steps of the TP, although separated by long time intervals, are effectively correlated. These results have been subsequently reproduced by means of different analytical techniques in Refs.[72,73] and [74].

As in the previous sections, we focus here on TP dynamics in the biased case, when the TP is charged, while the lattice gas particles are neutral, and the system is subject to a constant electric field acting on the TP only. We aim to determine exactly the TP mean-displacement $\left\langle\mathbf{R}_{n}\right\rangle$, which will give us an access to the TP mobility and thus will allow to verify the validity of the Einstein relation in this model with remarkably confined dynamics.

A standard approach to define the properties of the TP random walk, as exposed in Section 2, would be to start with a master equation determining the evolution of the whole configuration of particles. In doing so, similarly to the analysis of the tracer diffusion on $2 \mathrm{D}$ lattices in the presence of a finite vacancy concentration (see, e.g. Ref.[40]), one obtains the evolution of the joint distribution $P_{n}\left(\mathbf{R}_{n}, \mathbf{Y}\right)$ of the TP position $\mathbf{R}_{n}$ and of the vacancy position $\mathbf{Y}$ at time moment $n$. The property of interest, i.e. the reduced distribution function of the TP alone will then be found from $P_{n}\left(\mathbf{R}_{n}, \mathbf{Y}\right)$ by performing lattice summation over all possible values of the variable $\mathbf{Y}$.

Here we pursue, however, a different approach, which has been first put forward in the original work of Brummelhuis and Hilhorst [55]; that is, we construct the distribution function of the TP position at time $n$ directly in terms of the return probabilities of the random walk performed by the vacancy. The only complication, compared to the unbiased case considered by Brummelhuis and Hilhorst [55], is that in our case ten different return 
probabilities would be involved, instead of three different ones appearing in the unbiased case. Hence, the analysis will be slightly more involved.

We begin by introducing some basic notations. Let

- $P_{n}\left(\mathbf{R}_{n}\right)$ be the probability that the TP, which starts its random walk at the origin, appears at the site $\mathbf{R}_{n}$ at time moment $n$, given that the vacancy is initially at site $\mathbf{Y}_{0}$.

- $F_{n}^{*}\left(\mathbf{0} \mid \mathbf{Y}_{\mathbf{0}}\right)$ be the probability that the vacancy, which starts its random walk at the site $\mathbf{Y}_{0}$, arrives at the origin $\mathbf{0}$ for the first time at the time step $n$.

- $F_{n}^{*}\left(\mathbf{0}\left|\mathbf{e}_{\boldsymbol{\nu}}\right| \mathbf{Y}_{\mathbf{0}}\right)$ be the conditional probability that the vacancy, which starts its random walk at the site $\mathbf{Y}_{0}$, appears at the origin for the first time at the time step $n$, being at time moment $n-1$ at the site $\mathbf{e}_{\boldsymbol{\nu}}$.

Further on, for any time-dependent quantity $L_{n}$ we define the generating function of the form:

$$
L(\xi)=\sum_{n=0}^{+\infty} L_{n} \xi^{n}
$$

and for any space-dependent quantity $Z(\mathbf{X})$ the discrete Fourier transform

$$
\widetilde{Z}(\mathbf{k})=\sum_{\mathbf{X}} \exp (i(\mathbf{k} \cdot \mathbf{X})) Y(\mathbf{X})
$$

where the sum with the subscript $\mathbf{X}=\left(x_{1}, x_{2}\right)$ runs over all lattice sites.

Now, following Brummelhuis and Hilhorst [55], we write down directly the equation obeyed by the reduced probability distribution $P_{n}\left(\mathbf{R}_{n}\right)$ (cf Ref.[73] for a study of the joint probability of the TP position and of the vacancy position in the unbiased case):

$$
\begin{aligned}
P_{n}\left(\mathbf{R}_{n}\right) & =\delta_{\mathbf{R}_{n}, \mathbf{0}}\left(1-\sum_{j=0}^{n} F_{j}^{*}\left(\mathbf{0} \mid \mathbf{Y}_{\mathbf{0}}\right)\right)+ \\
& +\sum_{p=1}^{+\infty} \sum_{m_{1}=1}^{+\infty} \ldots \sum_{m_{p}=1}^{+\infty} \sum_{m_{p+1}=0}^{+\infty} \delta_{m_{1}+\ldots+m_{p+1}, n} \sum_{\nu_{1}} \ldots \sum_{\nu_{p}} \delta_{\mathbf{e}_{\nu_{1}}+\ldots+\mathbf{e}_{\nu_{p}}, \mathbf{R}_{n}} \times \\
& \times\left(1-\sum_{j=0}^{m_{p+1}} F_{j}^{*}\left(\mathbf{0} \mid-\mathbf{e}_{\boldsymbol{\nu}_{\mathbf{p}}}\right)\right) \times \\
& \times F_{m_{p}}^{*}\left(\mathbf{0}\left|\mathbf{e}_{\boldsymbol{\nu}_{\mathbf{p}}}\right|-\mathbf{e}_{\boldsymbol{\nu}_{\mathbf{p}-1}}\right) \ldots F_{m_{2}}^{*}\left(\mathbf{0}\left|\mathbf{e}_{\boldsymbol{\nu}_{\mathbf{2}}}\right|-\mathbf{e}_{\boldsymbol{\nu}_{\mathbf{1}}}\right) F_{m_{1}}^{*}\left(\mathbf{0}\left|\mathbf{e}_{\boldsymbol{\nu}_{\mathbf{1}}}\right| \mathbf{Y}_{\mathbf{0}}\right) .
\end{aligned}
$$


Next, using the definition of the generating functions and of the discrete Fourier transforms, Eqs.(73) and (74), we obtain the following matricial representation of the generating function of the TP probability distribution:

$$
\widetilde{P}(\mathbf{k} ; \xi)=\frac{1}{1-\xi}\left(1+\mathcal{D}^{-1}(\mathbf{k} ; \xi) \sum_{\mu} U_{\mu}(\mathbf{k} ; \xi) F^{*}\left(\mathbf{0}\left|\mathbf{e}_{\boldsymbol{\mu}}\right| \mathbf{Y}_{\mathbf{0}} ; \xi\right)\right)
$$

In Eq.(76) the function $\mathcal{D}(\mathbf{k} ; \xi)$ stands for the determinant of the following $4 \times 4$ matrix,

$$
\mathcal{D}(\mathbf{k} ; \xi) \equiv \operatorname{det}(\mathbf{I}-\mathbf{T}(\mathbf{k} ; \xi))
$$

where $\mathbf{T}(\mathbf{k} ; \xi)$ is also a $4 \times 4$ matrix with the elements:

$$
(\mathbf{T}(\mathbf{k} ; \xi))_{\nu, \mu}=\exp \left(i\left(\mathbf{k} \cdot \mathbf{e}_{\boldsymbol{\nu}}\right)\right) A_{\nu,-\mu}(\xi)
$$

which is given explicitly by

$$
\mathbf{T}(\mathbf{k} ; \xi) \equiv\left(\begin{array}{cccc}
e^{i k_{1}} A_{1,-1}(\xi) & e^{i k_{1}} A_{1,1}(\xi) & e^{i k_{1}} A_{1,-2}(\xi) & e^{i k_{1}} A_{1,2}(\xi) \\
e^{-i k_{1}} A_{-1,-1}(\xi) & e^{-i k_{1}} A_{-1,1}(\xi) & e^{-i k_{1}} A_{-1,-2}(\xi) & e^{-i k_{1}} A_{-1,2}(\xi) \\
e^{i k_{2}} A_{2,-1}(\xi) & e^{i k_{2}} A_{2,1}(\xi) & e^{i k_{2}} A_{2,-2}(\xi) & e^{i k_{2}} A_{2,2}(\xi) \\
e^{-i k_{2}} A_{-2,-1}(\xi) & e^{-i k_{2}} A_{-2,1}(\xi) & e^{-i k_{2}} A_{-2,-2}(\xi) & e^{-i k_{2}} A_{-2,2}(\xi)
\end{array}\right)
$$

and the coefficients $A_{\nu, \mu}(\xi), \nu, \mu= \pm 1, \pm 2$, defined as

$$
A_{\nu, \mu}(\xi) \equiv F^{*}\left(\mathbf{0}\left|\mathbf{e}_{\boldsymbol{\nu}}\right| \mathbf{e}_{\boldsymbol{\mu}} ; \xi\right)=\sum_{n=0}^{+\infty} F_{n}^{*}\left(\mathbf{0}\left|\mathbf{e}_{\boldsymbol{\nu}}\right| \mathbf{e}_{\boldsymbol{\mu}}\right) \xi^{n}
$$

are the generating functions of the conditional probabilities for the first time visit of the origin by the vacancy, Lastly, the matrix $U_{\boldsymbol{\mu}}(\mathbf{k} ; \xi)$ in Eq. $(76)$ is given by

$$
U_{\mu}(\mathbf{k} ; \xi) \equiv \mathcal{D}(\mathbf{k} ; \xi) \sum_{\nu}\left(1-e^{-i\left(\mathbf{k} \cdot \mathbf{e}_{\nu}\right)}\right)(\mathrm{I}-\mathrm{T}(\mathbf{k} ; \xi))_{\nu, \mu}^{-1} e^{i\left(\mathbf{k} \cdot \mathbf{e}_{\mu}\right)}
$$

The property of interest - the TP probability distribution function, will be then obtained by inverting $\widetilde{P}(\mathbf{k} ; \xi)$ with respect to the wave-vector $k$ and to the variable $\xi$ :

$$
P_{n}\left(\mathbf{R}_{n}\right)=\frac{1}{2 i \pi} \oint_{\mathcal{C}} \frac{\mathrm{d} \xi}{\xi^{n+1}} \frac{1}{(2 \pi)^{2}} \int_{-\pi}^{\pi} \mathrm{d} k_{1} \int_{-\pi}^{\pi} \mathrm{d} k_{2} e^{i\left(\mathbf{k} \cdot \mathbf{R}_{n}\right)} \widetilde{P}(\mathbf{k} ; \xi),
$$


where the contour of integration $\mathcal{C}$ encircles the origin counterclockwise.

After straightforward but rather tedious calculations (see, Ref.[76]), we find that in the small-k limit and $\xi \rightarrow 1^{-}$, which defines the leading large- $n$ behavior of the probability distribution, the generating function $\widetilde{P}(\mathbf{k} ; \xi)$ obeys

$$
\widetilde{P}(\mathbf{k} ; \xi)=\frac{1}{1-\xi}\left\{1-\left(-i \alpha_{0} k_{1}+\frac{1}{2} \alpha_{1} k_{1}^{2}+\frac{1}{2} \alpha_{2} k_{2}^{2}\right) \ln (1-\xi)\right\}^{-1}
$$

where the coefficients

$$
\left\{\begin{array}{l}
\alpha_{0}(E) \equiv \pi^{-1} \sinh (\beta E / 2)((2 \pi-3) \cosh (\beta E / 2)+1)^{-1} \\
\alpha_{1}(E) \equiv \pi^{-1} \cosh (\beta E / 2)((2 \pi-3) \cosh (\beta E / 2)+1)^{-1} \\
\alpha_{2}(E) \equiv \pi^{-1}(\cosh (\beta E / 2)+2 \pi-3)^{-1}
\end{array}\right.
$$

are all functions of the field strength $E$ and of the temperature only.

Now, the leading large- $n$ asymptotical behavior of the TP mean displacement can be obtained directly from Eq.(83), since the generating function of the TP mean displacement:

$$
<\mathbf{R}(\xi)>\equiv \sum_{n=0}^{+\infty}<\mathbf{R}_{n}>\xi^{n}
$$

obeys (see, e.g. Ref.[51]):

$$
<\mathbf{R}(\xi)>=-i\left(\frac{\partial \widetilde{P}}{\partial k_{1}}(\mathbf{0} ; \xi) \mathbf{e}_{\mathbf{1}}+\frac{\partial \widetilde{P}}{\partial k_{2}}(\mathbf{0} ; \xi) \mathbf{e}_{\mathbf{2}}\right)
$$

Consequently, differentiating the expression on the right-hand-side side of Eq.(83) with respect to the components of the wave-vector $\mathbf{k}$, we find that the asymptotical behavior of the generating function of the TP mean displacement in the vicinity of $\xi=1^{-}$follows

$$
<\mathbf{R}(\xi)>\sim\left(\frac{\alpha_{0}(E)}{1-\xi} \ln \frac{1}{1-\xi}\right) \mathbf{e}_{1}
$$

Further on, using the discrete Tauberian theorem (cf. Ref.[69]) and Eq.(84), we find the following force-velocity relation for the system under study

$$
<\mathbf{R}_{n}>\sim\left(\alpha_{0}(E) \ln n\right) \mathbf{e}_{\mathbf{1}}=\left(\frac{1}{\pi} \frac{\sinh (\beta E / 2)}{(2 \pi-3) \cosh (\beta E / 2)+1} \ln n\right) \mathbf{e}_{\mathbf{1}}, \quad \text { as } n \rightarrow \infty,
$$


which shows that the TP mean displacement grows logarithmically with $n$. In the limit $\beta E \ll 1$, the coefficient $\alpha_{0}(E)$ obeys

$$
\alpha_{0}(E)=\frac{\beta E}{4 \pi(\pi-1)}+\mathcal{O}\left(E^{3}\right)
$$

and hence, the mobility $\mu_{n}$, defined in Eq.(3), follows

$$
\mu_{n} \sim \frac{\beta}{4 \pi(\pi-1)} \frac{\ln (n)}{n}, \quad \text { as } n \rightarrow \infty
$$

Comparing next the result in Eq.(90) with that for the diffusivity $D_{n}$, Eq.(72), derived by Brummelhuis and Hilhorst [55] for the unbiased case, we infer that the TP mobility and diffusivity do obey, at least in the leading in $n$ order, the generalized Einstein relation in Eq.(5)! Note, that this can not be, of course, an a priori expected result, in view of an intricate nature of the random walks involved and anomalous, logarithmic confinement of the random walk trajectories.

We turn next to calculation of the asymptotic forms of the probability distribution

$P_{n}\left(\mathbf{R}_{n}\right)$. Inverting $\widetilde{P}(\mathbf{k} ; \xi)$ with respect to $\mathbf{k}$, we find that in the large- $n$ and large- $X$ limits, in the general case when $0 \geq E<\infty, P_{n}\left(\mathbf{R}_{n}\right)$ obeys

$$
P_{n}\left(\mathbf{R}_{n}\right) \sim\left(\pi \sqrt{\alpha_{1}(E) \alpha_{2}(E)} \ln (n)\right)^{-1} \exp \left(\frac{\alpha_{0}(E)}{\alpha_{1}(E)} x_{1}\right) K_{0}\left(\eta_{E}(n)\right)
$$

where $K_{0}$ is the modified Bessel (McDonald) function of zeroth order, while

$$
\eta_{E}(\lambda) \equiv \sqrt{\frac{2}{\ln (\lambda)}+\frac{\alpha_{0}^{2}(E)}{\alpha_{1}(E)}} \sqrt{\frac{x_{1}^{2}}{\alpha_{1}(E)}+\frac{x_{2}^{2}}{\alpha_{2}(E)}}
$$

$x_{1}$ and $x_{2}$ being the components of the vector $\mathbf{R}_{n}$. The distribution in Eq.(91) reduces to the result obtained by Brummelhuis and Hilhorst [55] in the limit $E=0$.

\section{Conclusions.}

To conclude, in this presentation we have overviewed some recent results on biased tracer diffusion in disordered dynamical environments represented here by hard-core lattice gases. We have considered several possible situations including 1D lattice gases with conserved 
and non-conserved particles density with initial homogeneous or "shock"-like particle distributions, $d$-dimensional lattice gases undergoing continuous exchanges with a reservoir, as well as an extreme case of biased tracer diffusion in a two-dimensional highly-packed lattice gas containing only a single vacancy. The main emphasis of our analysis here has been put on the question whether the Einstein relation between the tracer particle mobility and the diffusivity is valid, despite the fact that in some cases the tracer diffusion is anomalously confined or, in case of regular diffusion, the mobility and the diffusivity are non-trivial functions of the lattice gas density and other pertinent parameters. For some situations we were able to furnish an exact solution, which shows explicitly that this fundamental relation holds. In other instances, to calculate the tracer particle mobility we invoked an approximate approach based on decoupling of the third-order correlation functions. The tracer particle diffusivity has been then obtained assuming that the Einstein relations holds. Extensive numerical simulations have been performed, which confirm our analytical predictions and thus the validity of the Einstein relation.

Apart of it, we have shown that the lattice gas particles distribution as seen from moving TP is strongly inhomogeneous: the local particle density in front of the TP is higher than the average, which means that the lattice gas particles tend to accumulate in front of the driven TP, creating a sort of a "traffic jam", which impedes its motion. The condensed, "traffic jam"-like region in the case when the particles density is not explicitly conserved is characterized by a stationary density profile which vanishes as an exponential function of the distance from the tracer. The characteristic length and the amplitude of the density relaxation function have been calculated explicitly. In one dimensional situations with conserved particles density the "traffic jam" region grows in size in proportion to the TP mean displacement and no stationary profile exists. On the other hand, past the TP the local density is lower than the average. In one dimensional situations with conserved particle density this depleted by particles region also grows in size in proportion to the TP mean displacement. In higher dimensions, we have observed that depending on whether the number of particles in the is explicitly conserved or not, the local density past the TP may tend to the average value at large separations from the TP in a completely different 
fashion: In the non-conserved case the decay of the density is described by an exponential function, while for the conserved particles number case it shows an algebraic dependence on the distance, revealing in the latter case especially strong memory effects and strong correlations between the particle distribution in the monolayer and the tracer position. Further on, we have found that the terminal velocity $V$ of the TP depends explicitly on both the excess density in the "jammed" region in front of the TP, as well as on the density in the depleted region past the tracer. We realize that both densities are themselves dependent on the magnitude of the tracer velocity, applied external force, as well as on the rate of the adsorption/desorption processes and on the rate at which the particles can diffuse away of the tracer, which results in effective non-linear coupling between $V$ and $E$. In consequence, in the general case (for arbitrary adsorption/desorption rates and arbitrary external force), $V$ can be found only implicitly, as the solution of a transcendental equation relating $V$ to the system parameters. This equation simplifies considerably in the limit of a vanishingly small external bias; in this case we recover a linear force-velocity relation, akin to the so-called Stokes formula. This linear relation signifies that the frictional force exerted on the tracer particle by the host medium (the adsorbed monolayer) is viscous. The TP mobility, which is inverse of the Stokes friction coefficient, thus results from an intricate cooperative behavior.

\section{References}

[1] S.Havlin and D.Ben-Avraham, Advan. Phys. 36, 695 (1987)

[2] J.P.Bouchaud, A.Comtet, A.Georges and P.Le Doussal, Ann. Phys. (NY) 201, 285 (1990)

[3] J.P.Bouchaud and A.Georges, Phys. Repts. 195, 129 (1991)

[4] J.Klafter, R.Rubin and M.Shlesinger, eds., Transport and Relaxation in Random Media, (World Scientific, Singapore, 1986) 
[5] J.Drake and J.Klafter, Dynamics in Confined Molecular Systems, Physics Today 43, $46(1990)$

[6] J.Drake, J.Klafter and P.Levitz, Chemical and Biological Microstructures as Probed by Dynamic Processes, Science 251, 1574 (1991); J. M. Drake, P. Levitz, J. Klafter, N.J. Turro, K.S. Nitsche and K.F. Cassidy, Phys. Rev. Letters 61, 865 (1988)

[7] J. M. Drake, P. Levitz and J. Klafter, Time Resolved Studies of Optical Probes in Confining Spaces, in: "Dynamical Processes in Condensed Molecular Systems" Eds. J. Klafter, J. Jortner and A. Blumen (World Scientific, Singapore, 1989)

[8] S.F.Burlatsky, G.Oshanin and A.I.Chernoutsan, in: Low Dimensional Conductors and Superconductors, Springer Research Reports in Physics, eds. A.Ovchinnikov and I.Ukrainsky (Springer, Berlin, 1991), p.121

[9] G.Oshanin, M.Moreau and S.F.Burlatsky, Adv. Colloid and Interface Sci. 49, 1 (1994)

[10] G.Oshanin and S.F.Burlatsky, J. Stat. Phys. 65, 1109, 1095 (1991)

[11] A.Blumen, Nuovo Cimento B 63, 50 (1981)

[12] A.Blumen, J.Klafter and G.Zumofen, in: Optical Spectroscopy of Glasses, ed. I.Zschokke (Reidel, Dordrecht, 1986), p.199

[13] S.F.Burlatsky, G.Oshanin and A.Chernoutsan, Phys. Lett. A 149, 47 (1990)

[14] M.F.Shlesinger, H.Scher and J.G.Bendler, Phys. Today 1, 26 (1991)

[15] H.Scher and E.W.Montroll, Phys. Rev. B 12, 12, 2455 (1975)

[16] G.Oshanin, S.F.Burlatsky, M.Moreau and B.Gaveau, Chem. Phys. 177, 803 (1993)

[17] Y.Pomeau, A.Pumir and W.Young, Phys. Fluids A 1, 462 (1989)

[18] T.E.Harris, J. Appl. Prob. 2, 323 (1965)

[19] S.Alexander and P.Pincus, Phys. Rev. B 18, 2011 (1978) 
[20] T.M.Ligget, Interacting Particle Systems, (Springer, New York, 1985)

[21] M.J.A.M. Brummelhuis and H.J.Hilhorst, J. Stat. Phys. 53, 249 (1988); see also: Marco Brummelhuis, Application of Random Walk: Tracer Diffusion, Lattice Covering, and Damage Spreading, Ph. D. Thesis, Leiden University, The Netherlands, 1991

[22] Ya.Sinai, Theory Probab. Appl. 27, 247 (1982)

[23] H.Kesten, Physica A 138, 299 (1986)

[24] S.Havlin, J. Stat. Phys. 58, 653 (1990)

[25] J.Klafter, G.Zumofen and A.Blumen, Chem. Phys. 177, 821 (1993); M. F. Shlesinger, J. Klafter and G. Zumofen, Above, Below and Beyond Brownian Motion, American J. of Phys. 67, 1253 (1999)

[26] S.D.Druger, A.Nitzan, and M.A.Ratner, J. Chem. Phys. 79, 3133 (1983); S.D.Druger, M.A.Ratner, and A.Nitzan, Phys. Rev. B 31, 3939 (1985)

[27] A.K.Harrison and R.Zwanzig, Phys. Rev. A 32, 1072 (1985)

[28] R.Granek and A.Nitzan, J. Chem. Phys. 90, 3784 (1989)

[29] O.Bénichou, J.Klafter, M.Moreau and G.Oshanin, Phys. Rev. E 62, 3327 (2000)

[30] E.E.Polymeropoulos and J.Brickmann, Ann. Rev. Biophys. 14, 315 (1985)

[31] S.Chandra, Superionic Solids, Principles and Applications, (North-Holland, Amsterdam, 1981)

[32] S.Bhattarcharya, J.P.Stokes, M.W.Kim, and J.S.Huang, Phys. Rev. Lett. 55, 1884 $(1985)$

[33] G.S.Grest, I.Webman, S.A.Safran, and A.L.R.Bug, Phys. Rev. A 33, 2842 (1986)

[34] A.L.R.Bug and Y.Gefan, Phys. Rev. A 35, 1301 (1987) 
[35] C.M.C.Gambi, M.G.Giri, M.Carlà, D.Senatra, and A.Chittofrati, Phys. Rev. E 56, $4356(1997)$

[36] H.Cheradame, J.L.Souquet and J.M.Latour, Mater. Res. Bull. 15, 1173 (1980)

[37] B.L.Papke, R.Dupon, M.A.Ratner and D.F.Shriver, Solid State Ion. 5, 685 (1981)

[38] C.A.Vincent, Prog. Solid State Chem. 17, 145 (1987)

[39] P.Ferrari, S.Goldstein and J.L.Lebowitz, Diffusion, Mobility and the Einstein Relation, in: Statistical Physics and Dynamical Systems, eds.: J.Fritz, A.Jaffe and D.Szasz (Birkhäuser, Boston, 1985) p.405

[40] O.Bénichou, A.M.Cazabat, A.Lemarchand, M.Moreau and G.Oshanin, J. Stat. Phys. 97, $351(1999)$

[41] S.F.Burlatsky, G.Oshanin, M.Moreau and W.P.Reinhardt, Phys. Rev. E 54, 3165 (1996)

[42] C.Landim, S.Olla and S.B.Volchan, Commun. Math. Phys. 192, 287 (1998)

[43] G.Oshanin, J.De Coninck, M.Moreau and S.F.Burlatsky, in: Instabilities and NonEquilibrium Structures VII, ed. E Tirapegui, (Kluwer Academic Pub., Dordrecht), to be published; cond-mat/9910243

[44] Q.Gu, E.A.Schiff, S.Grebner and R.Schwartz, Phys. Rev. Lett. 76, 3196 (1996)

[45] F.Amblard, A.C.Maggs, B.Yurke, A.N.Pargellis and S.Leibler, Phys. Rev. Lett. 77, $4470(1996)$

[46] E.Barkai and J.Klafter, Phys. Rev. Lett. 81, 1134 (1998)

[47] E.Barkai and V.N.Fleurov, Phys. Rev. E 58, 1296 (1998)

[48] J.Kurchan, J. Phys. A 31, 3719 (1998)

[49] L.F.Cugliandolo, D.S.Dean and J.Kurchan, Phys. Rev. Lett. 79, 2168 (1997) 
[50] M.-C.Desjonquéres and D.Spanjaard, Concepts in Surface Physics, (Springer Verlag, Berlin, 1996)

[51] K.W.Kehr and K.Binder, in: Application of the Monte Carlo Method in Statistical Physics, ed. K.Binder, (Springer-Verlag, Berlin, 1987) and references therein.

[52] K.Nakazato and K.Kitahara, Prog. Theor. Phys. 64, 2261 (1980)

[53] R.A.Tahir-Kheli and R.J.Elliott, Phys. Rev. B 27, 844 (1983)

[54] H.van Beijeren and R.Kutner, Phys. Rev. Lett. 55, 238 (1985)

[55] M.Brummelhuis and H.Hilhorst, Physica A 156, 575 (1989)

[56] O.Bénichou, A.M.Cazabat, J.De Coninck, M.Moreau and G.Oshanin, Phys. Rev. Lett. 84, 511 (2000); Phys. Rev. B 63, 235413 (2001)

[57] S.F.Burlatsky, G.Oshanin, M.Mogutov and M.Moreau, Phys. Lett. A 166, 230 (1992)

[58] J.Crank, Free and Moving Boundary Problems, (Oxford University Press, Oxford, 1987)

[59] The authors wish to thank Professor J.L.Lebowitz for posing us this question and encouraging our analysis.

[60] D.B.Abraham, P.Collet, J.De Coninck and F.Dunlop, Phys. Rev. Lett. 65, 195 (1990)

[61] D.B.Abraham, P.Collet, J.De Coninck and F.Dunlop, J. Stat. Phys. 61, 509 (1990)

[62] J.De Coninck and F.Dunlop, J. Stat. Phys. 47, 827 (1987)

[63] G.Oshanin, A.M.Cazabat, J.De Coninck and M.Moreau, Phys. Rev. E 58, R20 (1998)

[64] G.Oshanin, A.M.Cazabat, J.De Coninck and M.Moreau, J. of Mol. Liquids 76, 195 (1998) 
[65] J.Lyklema, Solid-Liquid Interfaces, Fundamentals of Interface and Colloid Science, Vol. 2, (Academic-Harcourt-Brace, New York, 1995)

[66] R.Arratia, Z. Ann. Probab. 11, 362 (1983)

[67] R.F.Steiner, J. Chem. Phys 22, 1458 (1954)

[68] T.L. Hill, J. Chem. Phys 20, 1259 (1952)

[69] B.D.Hughes, Random Walks and Random Environments, (Oxford Science Publ., Oxford, 1995)

[70] P.Flajolet and A.M.Odlyzko, SIAM Journal on Discrete Math. 3, 216 (1990)

[71] Ajay and R.G.Palmer, J. Phys. A 23, 2139 (1990)

[72] T.J.Newman, Phys. Rev. B 59, 13754 (1999)

[73] Z.Toroczkai, Int. J. Mod. Phys. B11, 3343 (1997)

[74] R.K.P.Zia and Z.Toroczkai, J. Phys. A 31, 9667 (1998)

[75] M.J.A.M. Brummelhuis and H.J.Hilhorst, Physica A 156, 575 (1989)

[76] O.Bénichou and G.Oshanin, Phys. Rev. E 66, 031101 (2002) 